Check for updates

Cite this: Mater. Chem. Front. 2020, 4, 1930

Received 21st April 2020,

Accepted 8th May 2020

DOI: 10.1039/d0qm00255k

rsc.li/frontiers-materials

\section{Biosafety materials: an emerging new research direction of materials science from the COVID-19 outbreak}

\author{
Yingjie Yu, $\dagger^{\mathrm{abc}}$ Fanqiang $\mathrm{Bu}, \dagger^{\mathrm{d}}$ Huiling Zhou, ${ }^{\mathrm{ab}}$ Yongheng Wang, ${ }^{a}$ Jianxun Cui, ${ }^{\mathrm{e}}$ \\ Xing Wang, (D) ${ }^{d}$ Guohui $\mathrm{Nie}^{\mathrm{c}}$ and Haihua Xiao (D) *ab
}

\begin{abstract}
The coronavirus disease 2019 (COVID-19) pandemic is a serious biosafety event that is causing a severe impact on the global society and economy. Thus, the importance of biosafety is once again being valued worldwide. Due to the outbreak of COVID-19, most national governments have been encouraged to speed up the development of biosafety, which places higher requirements on researchers in biosafety and relevant fields. Many problems have been exposed due to the outbreak of COVID-19, including unavailability of effective drugs and vaccines, difficulty in fast or real-time virus detection, insufficient protective equipment, and shortage of transportation equipment for infected patients. To a certain extent, these biosafety problems are partly due to the limited biosafety-related research in materials science. Currently, the tremendous research efforts in materials science around the world have provided a wide variety of materials with peculiar properties to solve biosafety problems. This review attempts to give a perspective on how the development of novel materials can help scientists tackle the challenges in biosafety. Considering the importance of materials science in the biosafety field, it is urgent for us to officially propose the brand new concept of "biosafety materials", which can be a future scientific discipline that utilizes materials science and theory simultaneously to produce materials, related products, and equipment to solve biosafety problems. Herein, we have aimed to draw worldwide attention on the new discipline of biosafety materials and the active cooperation between materials scientists and biosafety-related scientists to propel its development.
\end{abstract}

\section{Biosafety: in the spotlight once again}

\subsection{The outbreak of COVID-19: a reconsideration of the importance of biosafety}

The rapid development of modern biotechnology and the process of economic globalization have resulted in a series of biosafety issues, such as the escape of genetically modified organisms, invasion of alien species, and the global outbreak of infectious diseases, which pose a huge threat to the diversity of species, ecological environment and human society. ${ }^{1}$ To date, the continued deterioration of the COVID-19 pandemic has severely affected the society and economic development around

\footnotetext{
${ }^{a}$ Beijing National Laboratory for Molecular Science, State Key Laboratory of Polymer Physical and Chemistry, Institute of Chemistry, Chinese Academy of Science, Beijing 100190, China. E-mail: hhxiao@iccas.ac.cn

${ }^{b}$ University of Chinese Academy of Science, Beijing 100049, China

${ }^{c}$ Institute of Translation Medicine, Shenzhen Second People's Hospital, the First Affiliated Hospital of Shenzhen University, Health Science Center, Shenzhen 518035, China

${ }^{d}$ Beijing Laboratory of Biomedical Materials, Beijing University of Chemical Technology, Beijing 100029, China

${ }^{e}$ HEBEI Flance Nanotechnology Co., Ltd., Dingzhou 073000, China

$\dagger$ These authors contributed equally to this work.
}

the world. ${ }^{2-4}$ In response to the unprecedented challenges from the COVID-19 pandemic, the Chinese government immediately decided to include biosafety in its national security system, which brings the concept of "biosafety" into the spotlight again. ${ }^{5}$

The anthrax envelope bioterrorism attack resulted in several people being infected after the event of September 11, 2001. , $^{6,7}$ In 2014, the occurrences of laboratory safety accidents such as the infection of Bacillus anthracis and H5N1 influenza virus were mainly due to poor awareness of biosafety. ${ }^{8-10}$ Furthermore, multiple consecutive reports of biosafety events have attracted international attention to biosafety issues. Currently, the outbreak of COVID-19 brings biosafety to the forefront of people's consciousness, which fully strengthens the necessity for the related scientific research in the field of biosafety. ${ }^{11,12}$ After the outbreak of COVID-19, most national governments have been encouraged to speed up the development of biosafety, which puts forward higher requirements for researchers in biosafety and relevant fields. ${ }^{13}$ Therefore, it is necessary to enhance our awareness toward biosafety and implement dynamic real-time detection, identification and tracking of biosafety issues such as environmental disasters and biological threats and carry out surveillance and control measures immediately. 


\subsection{The research areas of biosafety}

The concept of biosafety has already been defined by the scientific community. ${ }^{14-16}$ It refers to the prevention and control of hazards caused by biological risk factors such as biotechnology and pathogens. However, there are two easily confused concepts: biosafety and biosecurity. ${ }^{17}$ Biosafety is the prevention of the large-scale loss of biological integrity, focusing both on ecology and human health. It emphasizes the passive prevention and control of unintentionally induced biotechnology and microbial biological hazards. ${ }^{18}$ In contrast, biosecurity refers to proactive measures to prevent intentional biological hazards and often refers to areas such as national security, biological weapon control, epidemic prevention management, food security, and species invasion. ${ }^{19}$ Although the two words biosafety and biosecurity have some subtle differences in meaning, without special emphasis, biosafety is generally used. Therefore, herein, the term "biosafety" is used in all subsequent sections.

The research purpose of biosafety is taking effective measures against biological threats. As summarized in Fig. 1, the research areas of biosafety cover a wide range of topics, including controlling infectious diseases, monitoring biotechnology risks, ensuring laboratory biological safety, protecting biological resources, preventing invasion of alien species, defending against biological warfare and biological terrorist attacks.

\section{The proposal of biosafety materials science}

\subsection{Definition of biosafety materials science}

Materials science has shaped the world around us ever since the dawn of civilization. A variety of game changing materials with peculiar properties have been developed to achieve

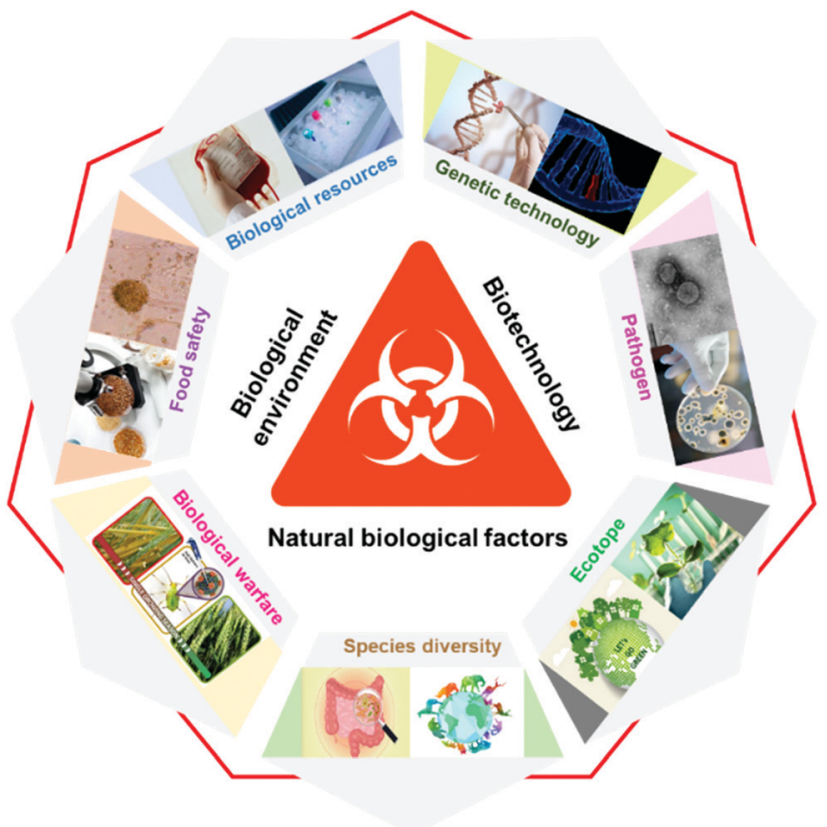

Fig. 1 Research areas of biosafety.

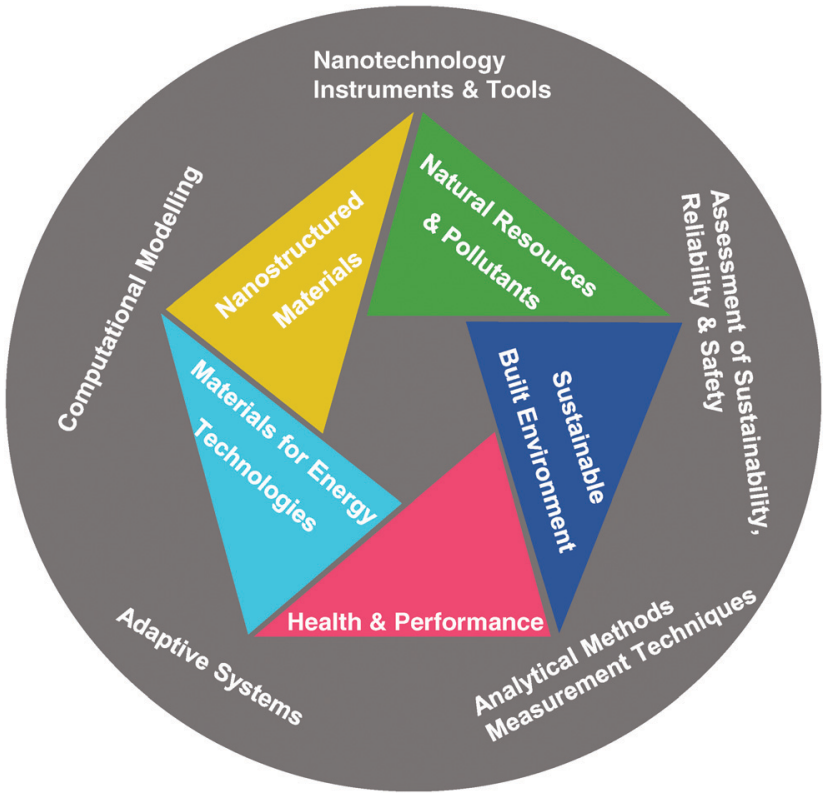

Fig. 2 Classification of materials.

unmet needs. $^{20-24}$ The Swiss Federal Laboratories for Materials Testing and Research (EMPA) briefly categorizes materials as nanostructured materials, materials for energy technology, materials for natural resources and pollutants, materials for health and performance, and materials for a sustainable built environment (Fig. 2). ${ }^{25}$ Over the past few decades, we have witnessed a revolution in materials science and how it has pushed forward the development of technology.

At this stage, the entire world is still experiencing a shortage of effective materials in response to biosafety threats. In the COVID-19 pandemic, the lack of biological safety personal protection equipment (PPE) such as masks, protective clothing, goggles, and negative pressure ambulance has resulted in a failure to protect numerous medical professionals. ${ }^{26-30}$ Moreover, insufficient sensitive and fast virus detection kits greatly impeded patients from being diagnosed, resulting in rapid spread of this pandemic. ${ }^{31,32}$ Hence, the marriage of biosafety with materials science will greatly help in resolving the existing challenges in biosafety fields, including the detection and disinfection of pathogens, viral vaccines, PPE and biological species preservation (Fig. 3). ${ }^{33-38}$ Therefore, here, we attempt to give a perspective on how the development of novel materials can help scientists tackle the challenges in biosafety. It is urgent and timely for us to officially propose the brand-new concept of "biosafety materials", which can be a future scientific discipline that utilizes materials science and theory simultaneously to produce materials, related products, and equipment to solve biosafety problems. To the best of our knowledge, the concept of "biosafety materials" as well as "biosafety material science" has never been officially proposed; therefore, the development of biosafety materials may still lack basic guiding ideology. As a result, researchers in materials science may not realize the problems and understand the challenges in biosafety, while researchers in biosafety may have no idea how materials can be applied to solve the biosafety 


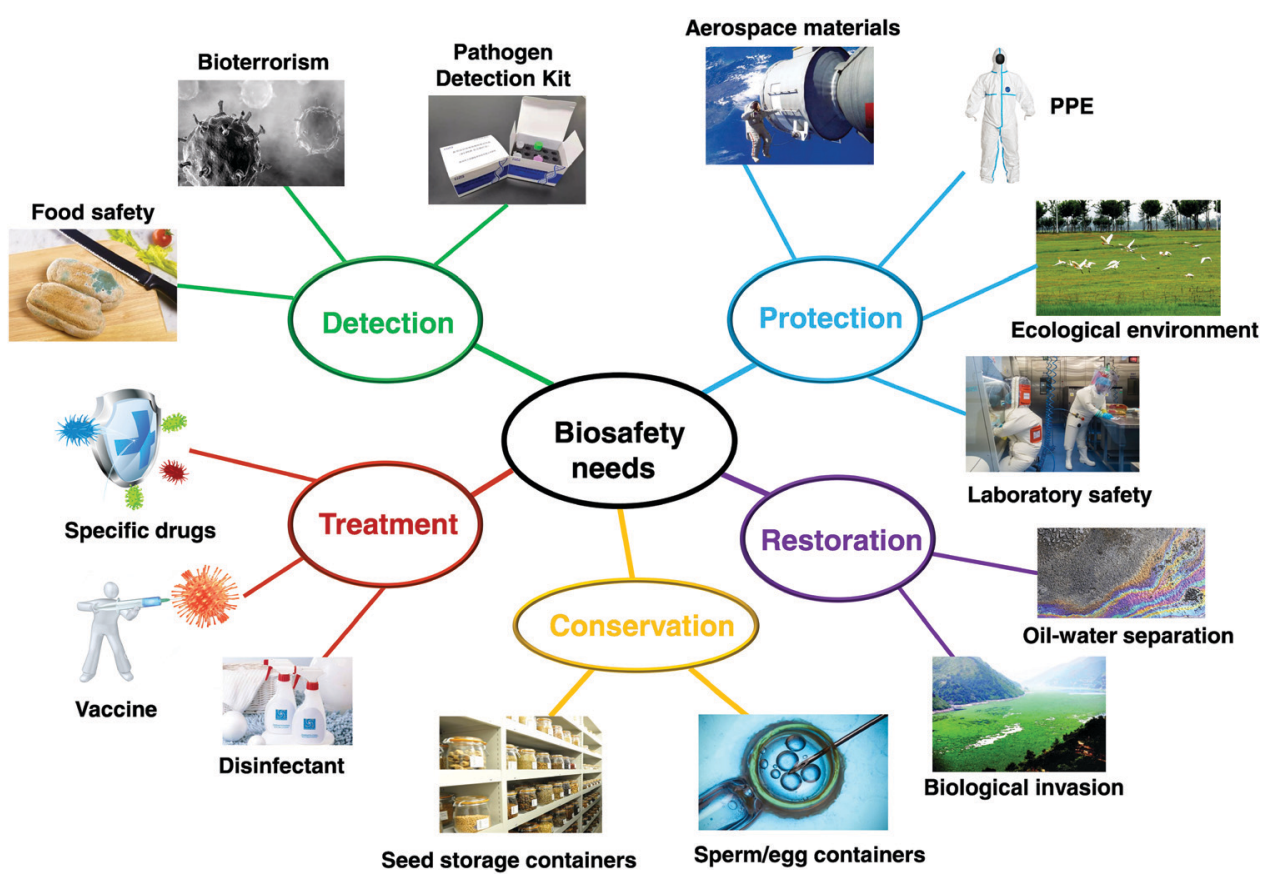

Fig. 3 Biosafety needs and their corresponding biosafety materials.

problems they face. Overall, it is of great significance and timely to put forward the brand-new concept of biosafety materials. By clarifying the concepts and role as well as the importance of biosafety materials, relevant researchers can work together to design advanced biosafety prevention and control materials, which will eventually improve our ability to tackle biosafetyrelated problems.

\subsection{Current challenges in the field of biosafety}

To address biosafety-related issues with materials, the current challenges in this field need to be specified. ${ }^{39-41}$ For example, the current mainstream pathogen detection method still largely relies on polymerase chain reaction (PCR), which is timeconsuming and laborious. Point-of-care testing (POCT) devices are highly desired due to their convenience. ${ }^{4-45}$ Luminescent materials provided a powerful tool to achieve sensitive and timely detection. ${ }^{46-48}$ Table 1 briefly summarizes the major challenges in biosafety from the perspective of materials science.

\section{3 "Biomaterials" and "biomedical materials"}

The term "biosafety materials" is also different from "biomaterials" and "biomedical materials". A biomaterial is any substance that has been engineered to interact with biological systems for a medical purpose, either a therapeutic (treat, augment, repair or replace a tissue function of the body) or diagnostic one. ${ }^{49-52}$ As a science, biomaterials is about fifty years old. The study of biomaterials is called biomaterials science or biomaterials engineering. It has experienced steady and strong growth over its history, with many companies investing large amounts of money into the development of new products. Biomaterials science encompasses elements of medicine, biology, chemistry, tissue engineering and materials science. ${ }^{53-55}$ Biomedical materials are biomaterials that are manufactured or processed to be suitable for use as medical devices (or components thereof), which are usually intended to be in long-term contact with biological materials. ${ }^{54,56}$ However, biosafety materials emphasize the use of materials for the prevention and control of biological safety issues. Taken together, biosafety materials are different from both "biomaterials" and "biomedical materials".

\section{4 "Biosafety materials" and "biosafety of materials"}

The biological safety of materials is mentioned frequently when people study biomaterials. ${ }^{57-59}$ Herein, considering that the concepts of "biosafety materials" and "biosafety of materials" are confusing, a comprehensive comparison between them is provided here. "Biosafety materials" in essence can be "materials for biosafety", which denote the application of materials and related theory to tackle biological safety issues. However, the latter "biosafety of materials" denotes whether materials are safe to biological systems, how toxicity occurs and to which extent there is toxicity. In general, the "biosafety" in the term "biosafety materials" refers to biological safety, namely, the biological parameters, such as virus and bacteria may cause safety issues to the environment and human body; however, the "biosafety" in the term "biosafety of materials" refers to the safety of certain materials to bio-organisms. The two biosafety terms are the same in written word, but they are terms used in different research fields from different backgrounds, and thus their meanings and connotations vary greatly.

Taking nanomaterials as an example, when the concept of nanomaterials was first proposed, most scientists were attracted by the fascinating properties nanomaterials brought with them, but few realized and considered their safety issues. In 2001, Prof. Yuliang Zhao from The National Center for Nanoscience 
Table 1 Current challenges in biosafety

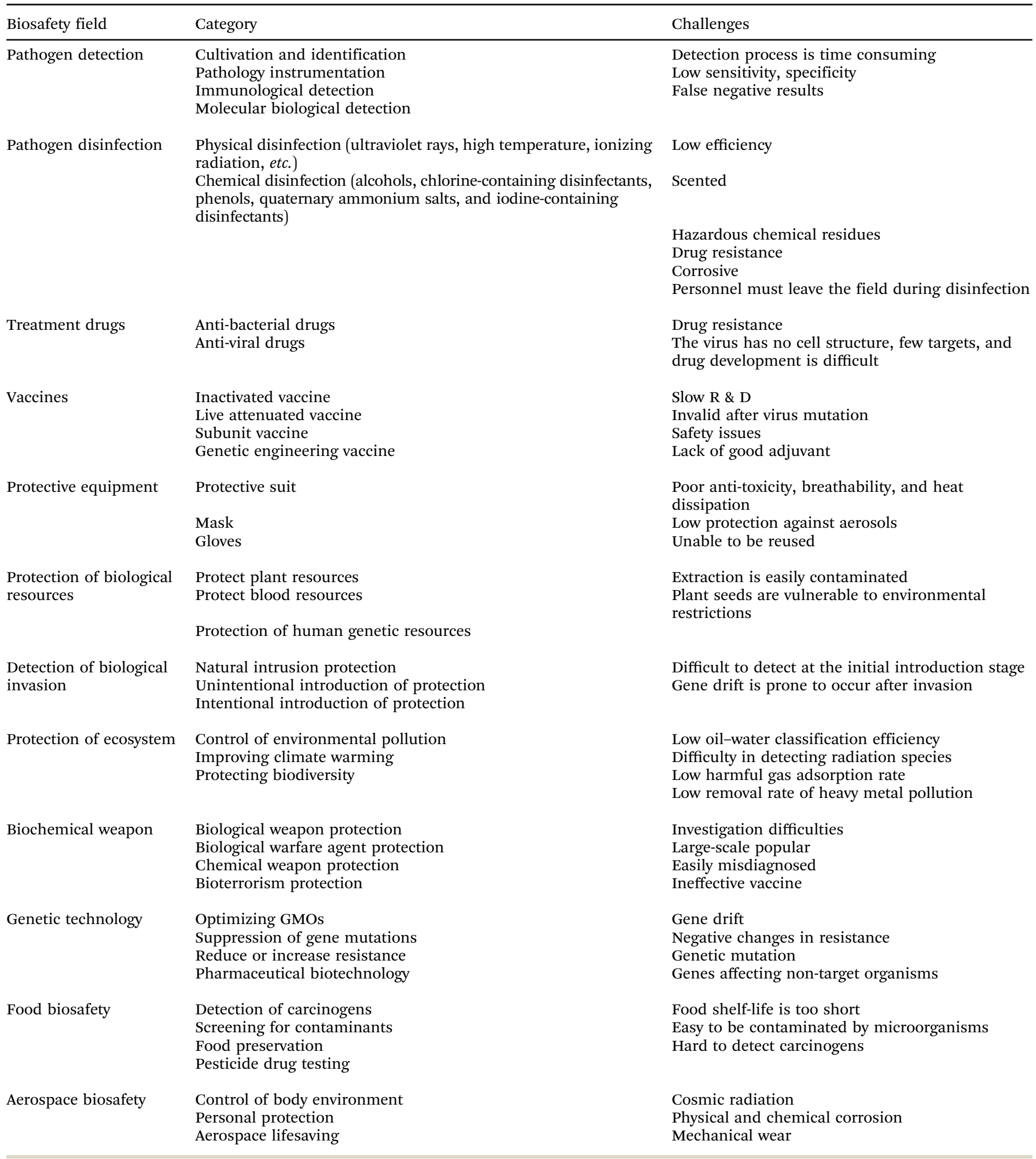

and Technology in China firstly proposed the concept "biosafety of nanomaterials", which is also termed nanotoxicity (nanosafety). ${ }^{60}$ Since then, the importance of the negative side (such as toxicity) of nanomaterials has been realized by scientists around the world. They further established the CAS (Chinese
Academy of Sciences) Key Laboratory for Biological Effects of Nanomaterials and Nanosafety in China. However, biosafety nanomaterials refer to the use of nanotechnology and nanomaterials to address biosafety issues, which is significantly different from the concept of nanotoxicity and biosafety of nanomaterials. To better 
Table 2 Biosafety materials and biosafety of materials

\begin{tabular}{lll}
\hline Item & Biosafety materials & Biosafety of materials \\
\hline Definition & $\begin{array}{l}\text { Develop materials for prevention and control biological } \\
\text { safety issues. }\end{array}$ & Evaluate the side effects and toxicity of materials. \\
Research content & $\begin{array}{l}\text { Design and develop new materials for biosafety issues; } \\
\text { evaluate their abilities for prevention and control of the } \\
\text { biological threat. }\end{array}$ & $\begin{array}{l}\text { Study the toxicity and toxicology of materials to bio- } \\
\text { logical species. }\end{array}$ \\
Research aim & $\begin{array}{l}\text { Protect human health and other species. } \\
\text { Conserve the biodiversity. } \\
\text { Protect the ecological environment. } \\
\text { Prevention and control other biological issues. }\end{array}$ & $\begin{array}{l}\text { Determine whether there is any toxicity of materials. } \\
\text { Understand to what extent the toxicity of materials is } \\
\text { and how the toxicity occurs. } \\
\text { Determine the possible acceptable dosage of materials }\end{array}$
\end{tabular}

Research direction

Find the most efficient, cheapest and best materials to solve biosafety issues.

Reduce the toxicity of materials to the human body.

understand this, these two terms, "biosafety of materials" and "biosafety materials", are compared in detail in Table 2.

\section{State-of-the-art of biosafety materials}

Although the concept of "biosafety material science" has not been officially proposed to date, we can still find a number of studies related to the biosafety materials, including pathogen detection, ${ }^{61-63}$ virus detection, ${ }^{64-68}$ prevention and control of infectious diseases, ${ }^{69-71} \mathrm{PPE},{ }^{72-75}$ preservation of biological and human resources, ${ }^{76-79}$ biological invasion monitoring, ${ }^{80-82}$ biological weapons and biological terrorist defense. ${ }^{83-87}$ Herein, the state-of-the-art biosafety materials are discussed in depth, which provides a comprehensive understanding for researchers in the related field and can help propel this concept in the newly emerging field.

\subsection{Biosafety materials for viral vaccines}

The long battle history between viruses and humans has proven that vaccination is the best solution to completely contain the spread of viruses. ${ }^{88-90}$ It effectively prevents disease through boosting the immune system against a pathogen. Since there are safety concerns on viral delivery systems, several biosafety materials have been extensively investigated for the in vivo delivery and controlled release of viral vaccines, including liposomes, polymers, cationic proteins and biological membranes. ${ }^{91,92}$ Viral vaccines can be delivered in the form of DNA, mRNA or protein, ${ }^{93}$ all of which can be easily enzymatically degraded upon entering the blood circulation. ${ }^{94,95}$ Taking SARS-CoV-2 for example, it contains 1273 amino acids with a molecular weight of about $140 \mathrm{kDa}$. The DNA encoding SARS-CoV-2 is more than $3800 \mathrm{bp}^{96}$ Thus, to protect the vaccine in circulation and help it be endocytosed into the cells, delivery vehicles are required. Moderna utilizes ionizable liposomes to carry negatively charged mRNA for the SARS-CoV-2 spike protein. ${ }^{97,98}$ A phase I clinical trial is underway in Seattle, (NCT04283461, USA). Moreover, research has shown that polymers such as low-molecularweight polyethyleneimine (PEI) modified with fatty chains and poly( $\beta$-amino) esters (PABEs) can be designed to deliver DNA and mRNA. ${ }^{92,99}$ In addition, protamine, as a natural cationic protein, can form complexes with negatively charged nucleic acids, and thereby be utilized to deliver mRNA-based therapeutics and stimulate an immune response. ${ }^{92}$ Furthermore, biological membranes such as red blood cell membranes and extracellular vesicles including exosomes, apoptotic bodies and microvesicles can be isolated and utilized for the delivery of biomolecule-based vaccines. ${ }^{100,101}$ Recently, the microneedle patch, as a novel drug delivery system, has attracted extensive scientific interest due to its excellent properties such as painless penetration and excellent therapeutic efficacy. It provides a highly efficient transdermal delivery system to create sophisticated devices with superior features for biomedical applications. Gambotto's group in the University of Pittsburgh developed the SARS-CoV-2 vaccine based on microneedle arrays (Fig. 4) ${ }^{102}$ Compared to the traditional subcutaneous needle injection, MNA SARS-CoV-2 subunit vaccines elicited strong and long-lasting antigen-specific antibody responses. In summary, biosafety materials are of great importance in the development of biomolecule-based therapeutics and are already used to combat viral infections.

\subsection{Biosafety materials for pathogen detection}

3.2.1 Biosafety materials for viral detection. The development of vaccines and drugs usually takes as long as several years, which means there is possibly no effective way to protect against infectious pathogenic microorganisms in the early stage of infectious disease outbreaks. ${ }^{103}$ Hence, the early detection of viruses is the key to combating the spread of infectious diseases. This is because the information from tests can help governments make public health decisions about measures for the control and prevention of an outbreak. Reverse transcriptase polymerase chain reaction (RT-PCR) has been widely used as a gold standard for virus detection. Although it can provide a reliable result, the process for detection is laborious and time-consuming. Normally, it can take several days or up to even one week to obtain results on an exceptional circumstance, which can cause the best timing to control the disease outbreak to be missed. Thus, rapid virus detection systems such as POCT, with high sensitivity and selectivity are highly desired in the clinic. Accordingly, emerging materials with peculiar properties provide new options to meet this unmet demand. 

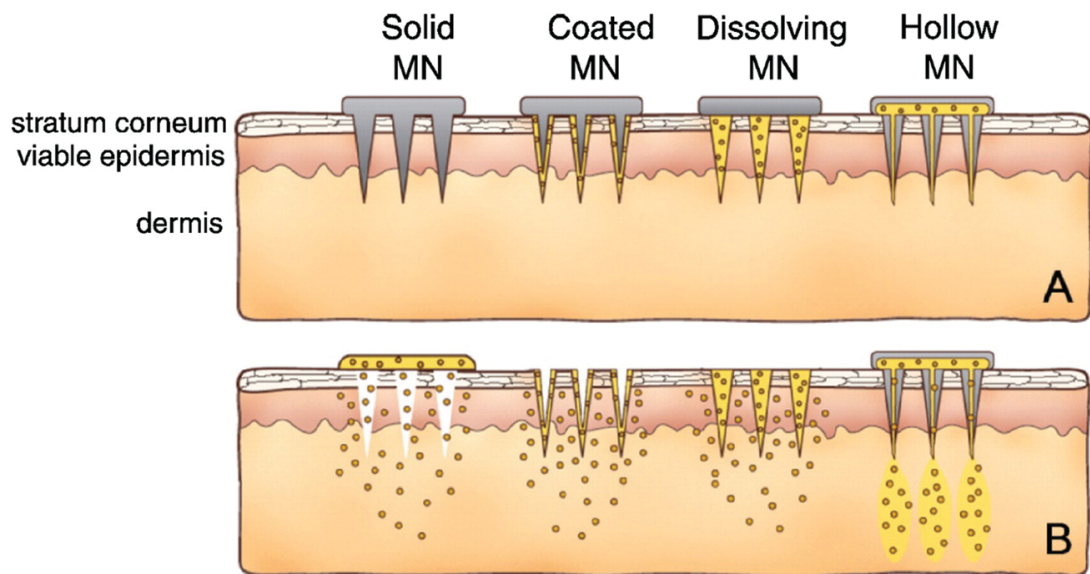

Fig. 4 Biosafety materials based on the microneedle patch for SARS-CoV-2 vaccine delivery. (A) Solid MNs pierce through the outer layers of the skin, leaving open space. (B) Vaccine diffuses into the skin through the open pores. Reproduced with permission from ref. 102. Copyright 2012, Elsevier.

Microfluidics is a revolutionary technology that manipulates small amounts of fluidics $\left(10^{-9}-10^{-18} \mathrm{~L}\right)$, thereby becoming powerful for viral detection (Fig. 5B). Yeh et al. reported a portable and high-throughput microfluidic VIRRION platform containing carbon nanotube arrays (Fig. 5A). ${ }^{104}$ VIRRION not only effectively captures different viruses by size, but also performs real-time nondestructive identification of virus using surface-enhanced Raman spectroscopy (SERS) coupled to a

A
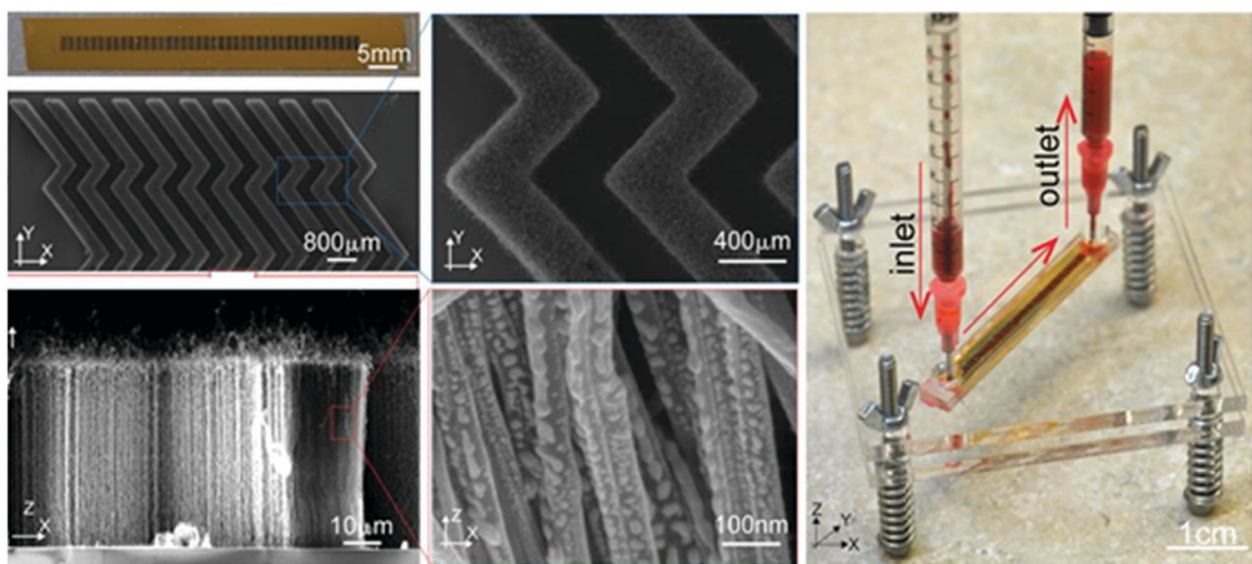

B
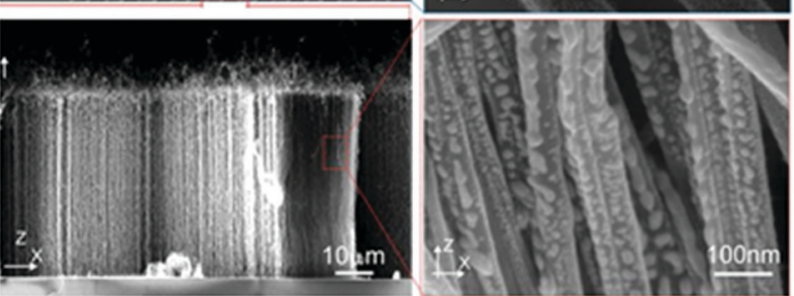

$\Leftrightarrow x$

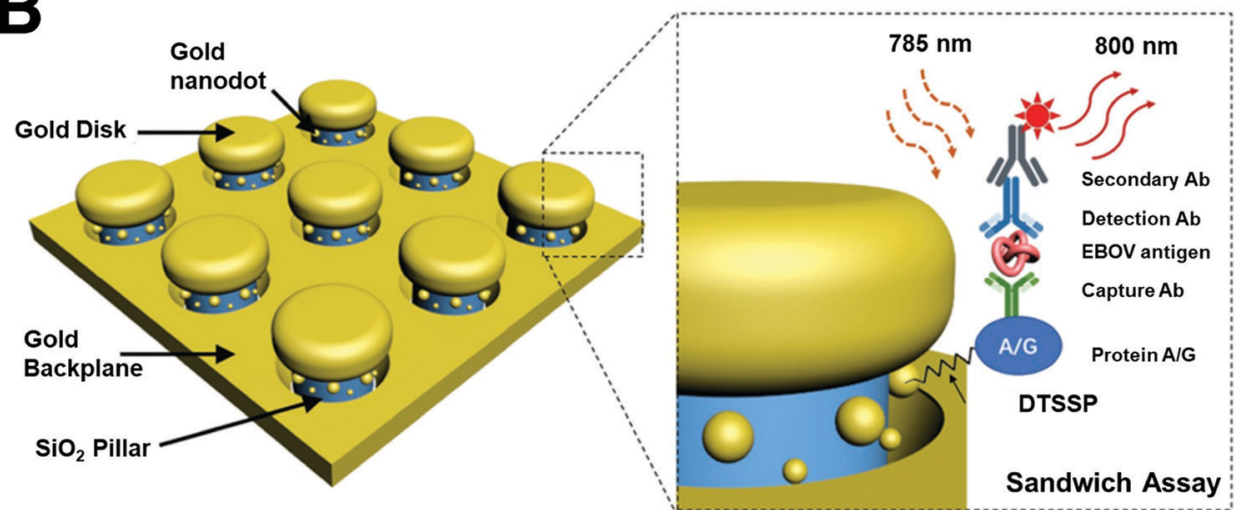

Fig. 5 Biosafety materials for virus capture and detection. (A) High-throughput microfluidic VIRRION platform containing carbon nanotube arrays for influenza virus capture and identification. Reproduced with permission from ref. 104. Copyright 2020, National Academy of Sciences. (B) Schematic illustration of 3D plasmonic nanoantenna array for EBOV sensor. Reproduced with permission from ref. 106. Copyright 2019, Wiley-VCH. 
machine learning and database. The research team validated this device using different subtypes of avian influenza A viruses and human samples with respiratory infections, reporting the successful enrichment of rhinovirus, influenza virus and parainfluenza viruses. This device is also reported to maintain stoichiometric viral proportions when samples contain more than one type of virus, suggesting it can also be functional in cases where coinfection has occurred. The processing time (including viral enrichment as well as detection) takes only a few minutes with a 70 -fold enrichment enhancement. The sensitivity of this system can reach as little as $10^{2}$ EID $_{50}$ per $\mathrm{mL}$, with a virus specificity of $90 \%$, indicating its potential to realize POCT. Furthermore, Sun et al. designed a point-of-care micro-fluid system integrated with a smartphone for live virus detection. The detection limit is comparable to the traditional RT-PCR, with the result achieved within 30 minutes. ${ }^{105}$ Hence, microfluid technology endows detection systems with appealing versatility and reliability, exhibiting distinct advantages over traditional RT-PCR.

The outbreak of the Ebola virus (EBOV) in West Africa underscored the need to develop highly sensitive tests for diagnosis as early as possible. Chou et al. developed a 3D plasma nanoantenna measurement sensor as an on-chip immunoassay platform for the ultra-sensitive detection of the EBOV antigen (Fig. 5B). ${ }^{106}$ Compared with the flat gold substrate, the EBOV sensor exhibited a significant increase in fluorescence intensity. The nano-antenna-based biosensor could detect EBOV soluble glycoprotein at a concentration as low as $220 \mathrm{fg} \mathrm{mL}^{-1}$. The sensitivity of this biosensor is 240000 times higher than the existing FDA (Food and Drug Administration)-recommended immunoassay-based tests. This sensor can be further adapted to a universal biosensing platform for other viruses. Zheng et al. utilized carbon nanotubes to develop a portable device that can selectively capture viruses based on their size. ${ }^{107}$ This carbon nanotube assay can selectively capture and aggregate viruses in diluted samples based on the size of the virus, thus increasing the detection threshold of the virus by a factor of 100 . During the whole isolation process, no antibody is required for detection, which simplifies the operation for viral detection.

Nanoenzymes, as functional nanomaterials with enzymelike characteristics, have gained tremendous attention in viral detection. ${ }^{108,109}$ For example, nanoenzymes demonstrated remarkable sensitivity and specificity in detecting the avian influenza A (H5N1) virus. Ahmed et al. utilized Au NP as peroxidase to amplify the signal for avian influenza A virus detection (Fig. 6A). ${ }^{110}$ This dual enhanced colorimetric immunosensor enabled the detection of $\mathrm{H} 5 \mathrm{~N} 1$ with a limit of detection (LOD) as low as $1.11 \mathrm{pg} \mathrm{mL}^{-1}$, suggesting that it was more sensitive than ELISA or bioassays based on plasmonics. It has been further applied to detect other avian influenza A viruses such as H4N6 and H9N2. In addition, Yan's group developed an $\mathrm{Fe}_{3} \mathrm{O}_{4}$ magnetic nanoparticle (MNP)based nanoenzyme-strip for the detection of EBOV, which is 100-fold more sensitive than the standard strip method, thereby providing a valuable simple screening tool for the diagnosis of various pathogens (Fig. 6B). ${ }^{111}$

3.2.2 Biosafety materials for bacteria detection. Most bacteria are harmless and even beneficial, but some bacteria are pathogens that can cause many infectious diseases such as tuberculosis, which result in about two million deaths each year. ${ }^{112,113}$ Moreover, the worldwide trend of the increasing prevalence of antimicrobial resistance resulting from the outright abuse of antibiotics has become a major public health problem. Thus, the development of POCT can significantly help identify an effective antibiotic for the infection of a patient. Xiao et al. developed a novel biosensor for the high-throughput detection of bacteria based on the DNA molecular machine. ${ }^{114}$ The platform successfully realized single-step, fast and multi-channel high-throughput bacterial detection, as shown in Fig. 7A. The SDwalker drop biosensor platform adopts the trigger spontaneous motion mechanism of a walking molecular machine, which has the characteristics of rapid analysis and single bacterial analysis. The signal amplification strategy based on SDwalker drop operation can
A

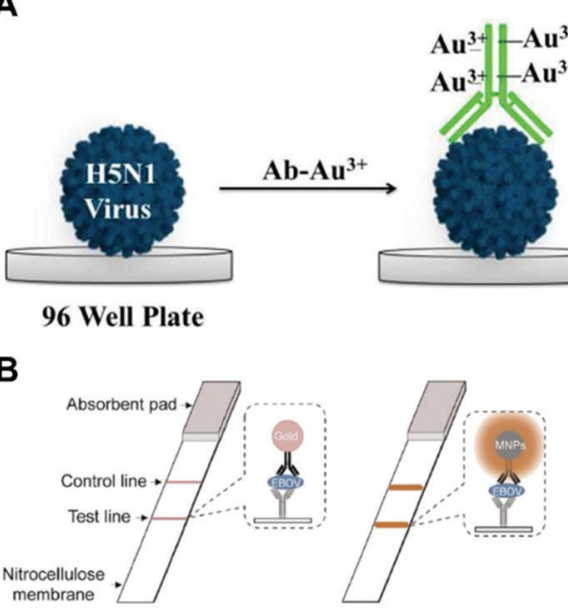

\section{TMBZ/H2O2}
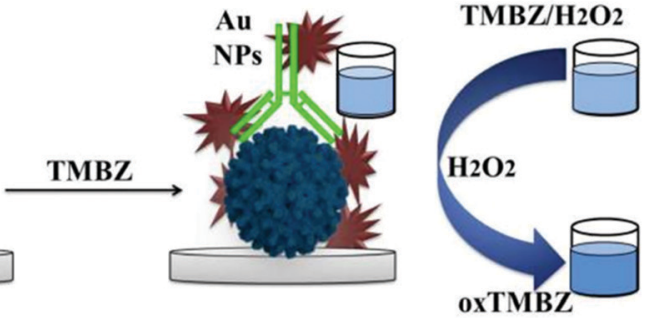

$\mathrm{EBOV}-\mathrm{GP}(\mathrm{ng} / \mathrm{mL})$
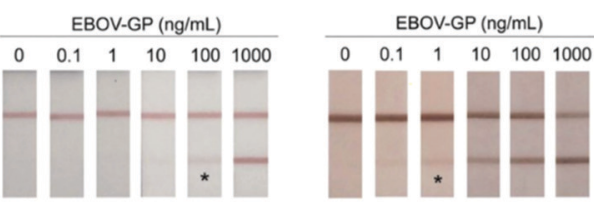

Fig. 6 Nanoenzyme-based biosafety materials applied in virus detection. (A) Mechanism of immunosensor with Au NPs for the detection of the avian influenza virus. Reproduced with permission from ref. 110. Copyright 2017, Ivyspring International Publisher. (B) Nanozyme strip based on MNP for the detection of EBOV. Reproduced with permission from ref. 111. Copyright 2015. Elsevier. 
A
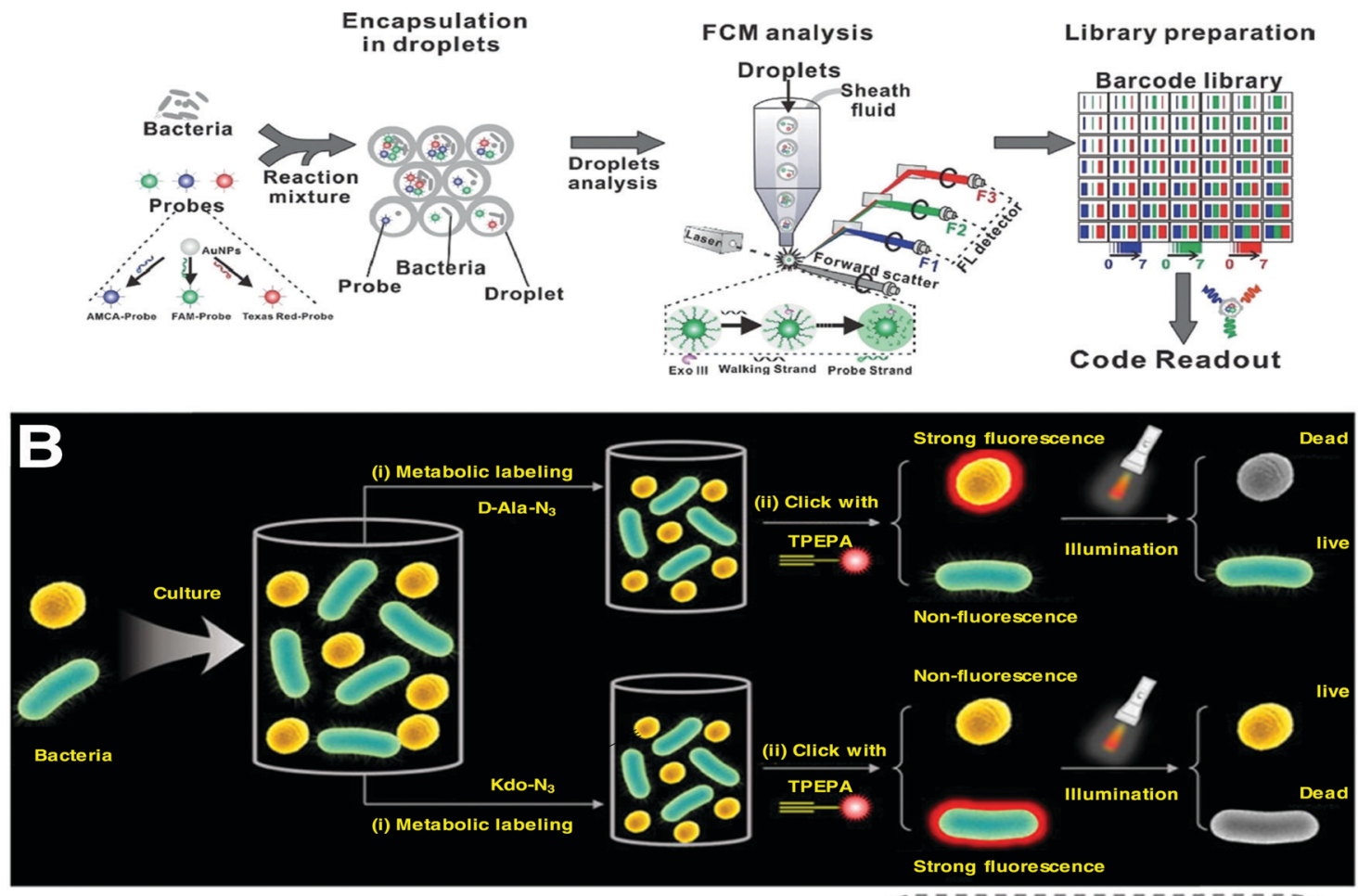

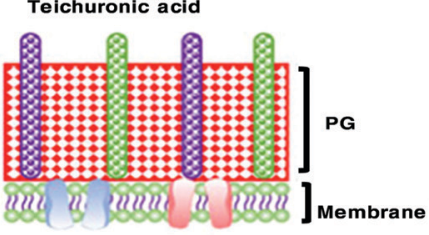

Gram-positive

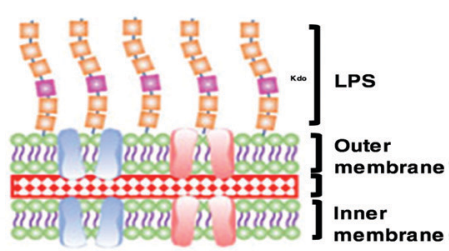

Gram-negative

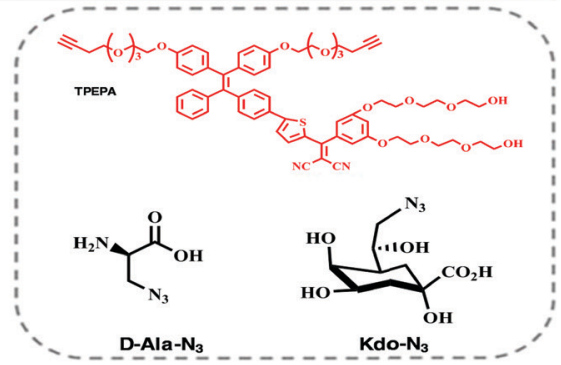

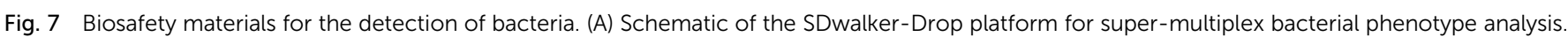

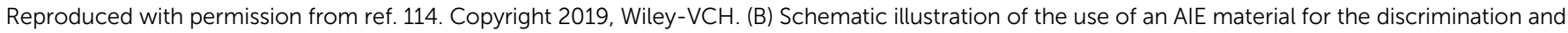
precise ablation of bacterial pathogens. Reproduced with permission from ref. 122. Copyright 2019, American Chemical Society.

be used for the highly sensitive detection of bacteria $\left(1 \mathrm{CFU} \mathrm{mL}{ }^{-1}\right)$, exhibiting a promising platform for the detection of bacteria.

Traditional fluorescent probes have the effect of aggregationinduced quenching (ACQ), but the complex physiological environment can reduce the selectivity and sensitivity of fluorescent probes. ${ }^{115,116}$ In contrast, molecules with the aggregationinduced emission (AIE) effect can overcome the shortcomings of traditional fluorescent molecules, and no complicated washing steps are required due to their property of low fluorescent background. ${ }^{117,118}$ These advantages of AIE molecules endow sensitivity and reliability to detection systems. ${ }^{119-121}$ Liu et al. designed a bioorthogonal fluorescence turn-on probe, TPEPA, for the discrimination and precise ablation of bacterial pathogens. ${ }^{122}$ TPEPA (tetraphenylene polyethylene glycol AIEgen) is a type of AIE photosensitizer with good water solubility, and its alkynyl group can be linked to an azide group via click reaction (Fig. 7B). Taking advantage of the difference in bacterial structures, TPEPA can discriminate pathogens via selective imaging of metabolically decorated Gram-negative bacteria with Kdo- $\mathrm{N}_{3}$ and Grampositive bacteria with $\mathrm{D}-\mathrm{Ala}-\mathrm{N}_{3}$, respectively, thus achieving the selective detection and killing of bacteria in situ. The use of the fluorescent properties of organic materials solves the problems of difficulty in accurately identifying pathogens in a short time. ${ }^{123-126}$

\subsection{Biosafety materials for disinfection}

The wide use of disinfection is beneficial in preventing infectious diseases, and thus is beneficial for the public health. It relies on physical or chemical methods to eliminate pathogens that stay on different transmission vehicles, thereby cutting off the transmission route to prevent and control the spreading of infections. Various chemical compounds such as alcohol, iodine-containing disinfectants, chlorine-containing disinfectants, peroxides, phenols, and quaternary ammonium salts have been widely used as disinfectant. However, these compounds suffer from multiple drawbacks such as harmfulness and corrosive nature. 
PAsp-b-PCL
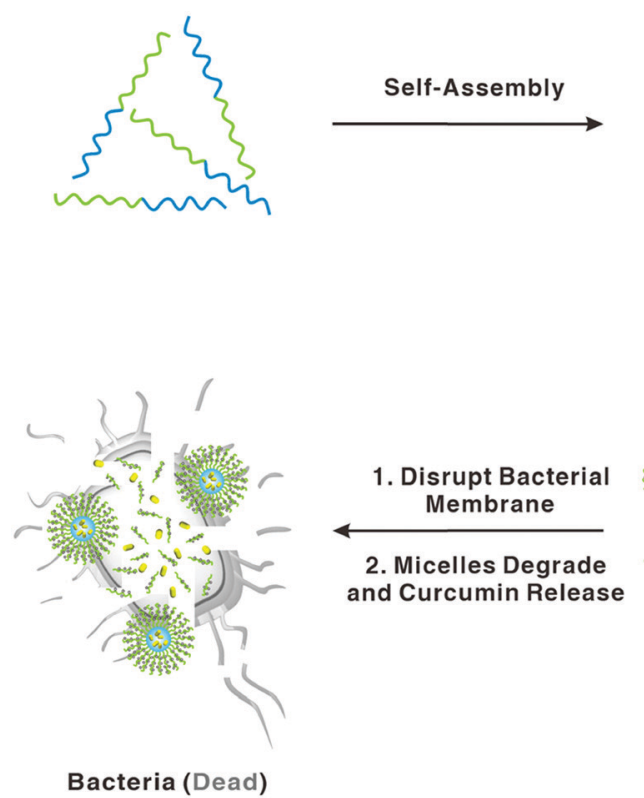
and

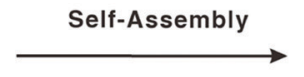

Polymeric Micelle (PM) and Curcumin Release

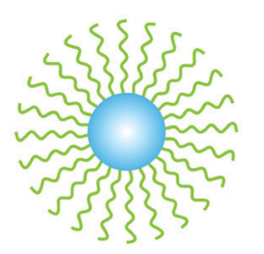

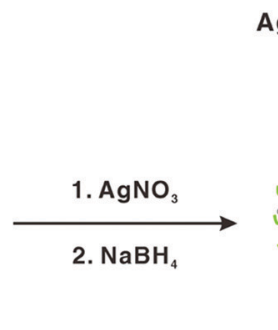

Ag-decorated Micelle (PM-Ag)

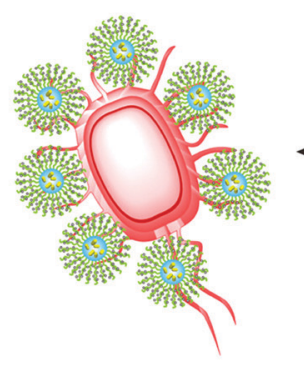

Bacteria (Active)
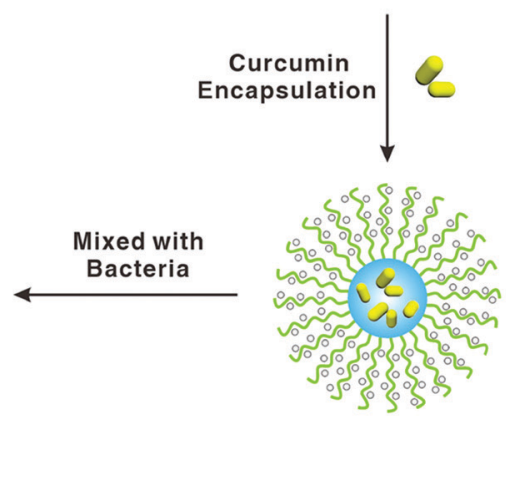

Curcumin-loaded Ag Micelle (PM-Ag-Cur)

Fig. 8 Biosafety materials based on Ag NPs for disinfection. Schematic illustration of the formation of silver-decorated polymeric micelles encapsulating curcumin simultaneously for enhanced antibacterial activity. Reproduced with permission from ref. 128. Copyright 2017, American Chemical Society.

Nanomaterials exhibit antimicrobial effects owing to their high surface-area-to-volume ratio and unique physical and chemical properties. Particularly, tremendous attention has been paid to Ag NPs due to their practical applications in our daily life. They have been widely used in different sectors such as silverbased air/water filters, textiles, animal husbandry, biomedical industry and food packaging. ${ }^{127}$ Huang et al. designed a novel polymeric micelle for simultaneously decorating Ag NPs and encapsulating curcumin as a combination strategy to improve the antibacterial efficiency (Fig. 8). ${ }^{128}$ Through rational design, the aggregation of Ag NPs could be avoided, and the solubility of curcumin was improved simultaneously. Excellent antibacterial activity toward the Gram negative P. aeruginosa and Gram positive $S$. aureus has been well demonstrated, thereby proving its potential in disinfection.

\subsection{Biosafety materials for drug treatment}

The discovery of anti-microbial agents has revolutionized our strategy to treat bacterial diseases in the twentieth century. ${ }^{129,130}$ However, the misuse and outright abuse of antibiotics have resulted in multidrug-resistant (MDR) pathogenic bacteria. Nanotechnology-based antibacterial strategies significantly enrich the tools to fight against MDR bacteria. As shown in Fig. 9A, Liang's group designed near infrared (NIR)-activated TRIDENT (Thermo-Responsive-Inspired Drug-Delivery Nano-Transporter) for effectively eradicating clinical methicillin-resistant Staphylococcus aureus. ${ }^{131}$ Imipenem (IMP, a broad-spectrum antibiotic) and IR 780 (a photosensitizer molecule) were encapsulated to fabricate smart triple-functional thermo-responsive nanoparticles. The temperature increase generated by NIR not only melted the nanotransporter via a phase change mechanism, but also damaged the bacterial membranes to facilitate imipenem permeation, thereby achieving robust bactericidal capabilities via chemo-photothermal therapy. The unique properties of carbonbased materials significantly aid in dealing with antimicrobial resistance. As shown in Fig. 9B, Zheng et al. designed an La@GO nanocomposite library for killing antibiotic-resistant bacteria. ${ }^{132}$ Unlike conventional antibiotics or $\mathrm{Ag}$, long-term exposure to La@GO at sub-MIC for 30 days did not induce detectable secondary resistance in E. coli. A novel extracellular multitarget invasion (EMTI) mechanism was also proposed to explain the result, which can help scientists develop more specific systems based on this system. The first antibiotic penicillin was discovered in 1928. Subsequently, numerous lives have been saved, and a variety of antibiotics have contributed to the control of infectious diseases. However, currently, there are very few antibiotics under development, and the number of effective antibiotics is declining. Thus, novel materials with versatile properties provide new strategies to treat MDR bacteria and viruses, providing a different route to overcome drug resistance.

\subsection{Biosafety materials for PPE}

Serving as the first line of defense in the fight against viruses, high-performance PPE is crucial for protecting frontline medical professionals and the general public. ${ }^{133,134}$ Current PPE suffers from poor light resistance, and no antimicrobial effect is provided. Furthermore, it is difficult for PPE materials to be effectively disinfected under the premise of good preservation. ${ }^{135,136}$ Hence, stable and protective materials with broad antimicrobial activity are in high demand. 
A

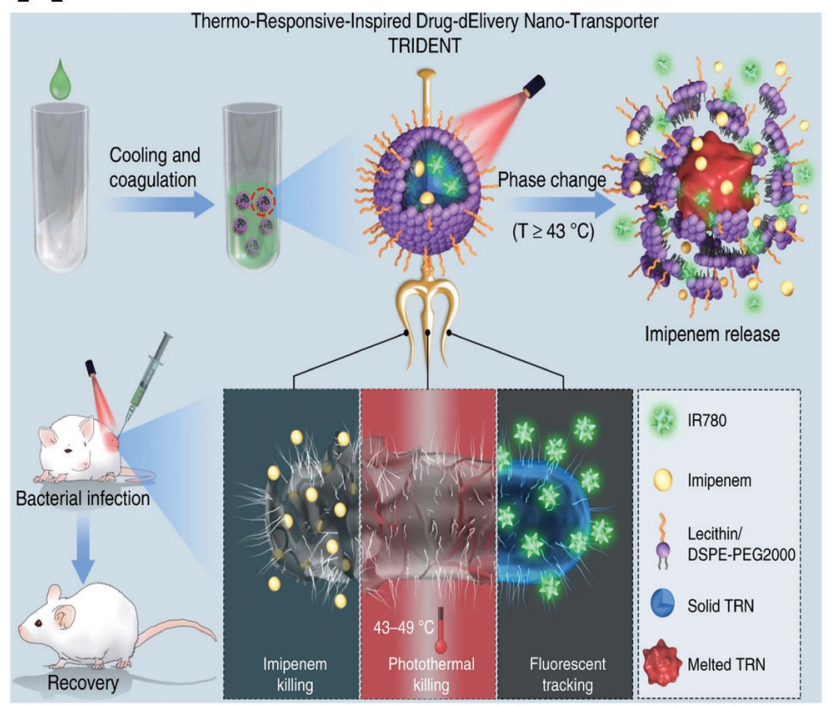

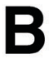
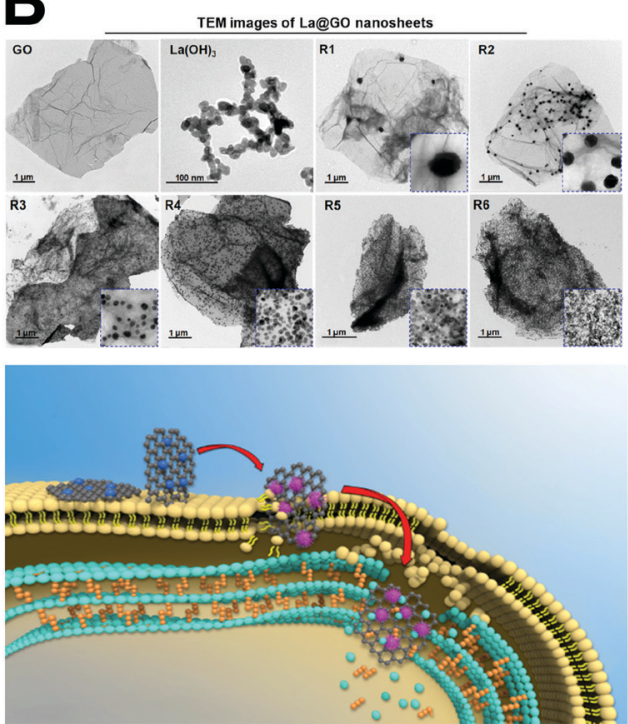

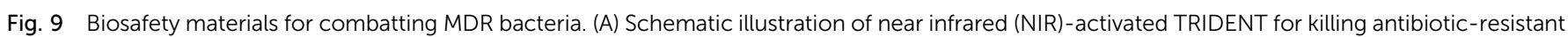

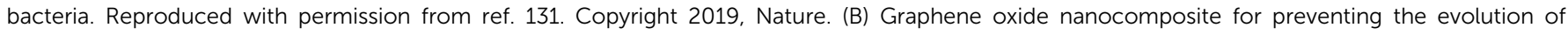
antimicrobial resistance. Reproduced with permission from ref. 132. Copyright 2019, American Chemical Society.

For stopping the spread of highly infectious diseases, air filtration has become an efficient passive pollution control strategy. Nevertheless, most of the commercial air purifiers solely rely on dense fibrous filters, which can effectively remove particulate matter but lack antibacterial activity. Li et al. designed a series of metalorganic frameworks (MOFs) with photocatalytic bactericidal properties to fabricate a nano-fiber membrane. ${ }^{137}$ It can effectively produce biocidal reactive oxygen species (ROS), which is driven by sunlight (Fig. 10A). Specifically, a zinc-imidazolate MOF (ZIF-8) exhibits almost complete inactivation of Escherichia coli $(E$. coli) (>99.9999\% inactivation efficiency) in saline within $2 \mathrm{~h}$ of simulated solar irradiation. This MOFilter provides new insights into the sustainable, self-charging, and adaptive development of protective materials, which represents the next generation of PPE. Moreover, a polymer material was developed to make face mask protection equipment (Fig. 10B). Liu et al. designed a novel self-powered electrostatic adsorption face mask (SEA-FM) based on a poly(vinylidene fluoride) electrospun nanofiber film (PVDFESNF) and a triboelectric nanogenerator (TENG). ${ }^{138}$ Up to $99.2 \%$ particulate removal efficiency could be achieved, which is much higher than that of the commercial mask. After the outbreak of COVID-19, the demand for masks has exploded worldwide. However, the disposal of the massive number of single-use masks poses a significant environmental threat to society. Thus, to ease this problem, reusable and recyclable graphene masks with outstanding superhydrophobic and photothermal performances were developed by Li et al. The high surface temperature of the masks under solar illumination can effectively sterilize the surface viruses. It is believed that more advance materials can be properly applied by scientists to produce multi-functional masks. ${ }^{135}$

Surgical suits are special clothing required by doctors to perform surgical operations. The materials used need to possess protective properties to block viruses and bacteria from invading medical personnel, which should not only be sterile, dust-free and disinfection resistance, but also isolate bacteria, antibacterial and comfortable. Accordingly, a three-layer antiviral surgical suit with non-woven spunbond polypropylene, polyester and microporous PTFE film was developed. ${ }^{139}$ Plasma technology was used to treat the outer layer of the spunbond polypropylene. The results showed that the plasma-treated surgical gown had a $99.04 \%$ reduction in microbes compared to the non-plasmatreated surgical gown, providing a microbial barrier for medical staff. $^{140-143}$

Respirators are a type of sanitary product, generally referring to that worn in the nose and mouth to filter the air entering the nose and mouth to block harmful gases, odors, droplets, viruses and other substances. Masks have a certain filtering effect on the air entering the lungs. When respiratory infectious diseases are prevalent and when working in a polluted environment such as a dusty area, wearing a mask is necessary to keep workers safe. ${ }^{144,145}$ Novel material fabrication technology such as $3 \mathrm{D}$ printing has been applied to manufacture masks. ${ }^{146}$ The N95 filtering facepiece respirator was custom designed for medical staff. Comfort and fit are essential while wearing an FFR, especially for medical professionals who need to work for a long period to treat patients. The face seal prototypes of masks were prepared with acrylonitrile butadiene styrene (ABS) plastic using 3D printing, which provide improved contact pressure for users.

\subsection{Biosafety materials for protection and preservation of biological resources}

With economic globalization, environmental pollution and global warming, biodiversity has faced unprecedented challenges. The preservation of biological resources provides a guarantee for the biodiversity of the ecological system. Generally, biological genetic resources mainly include the extraction and protection of 


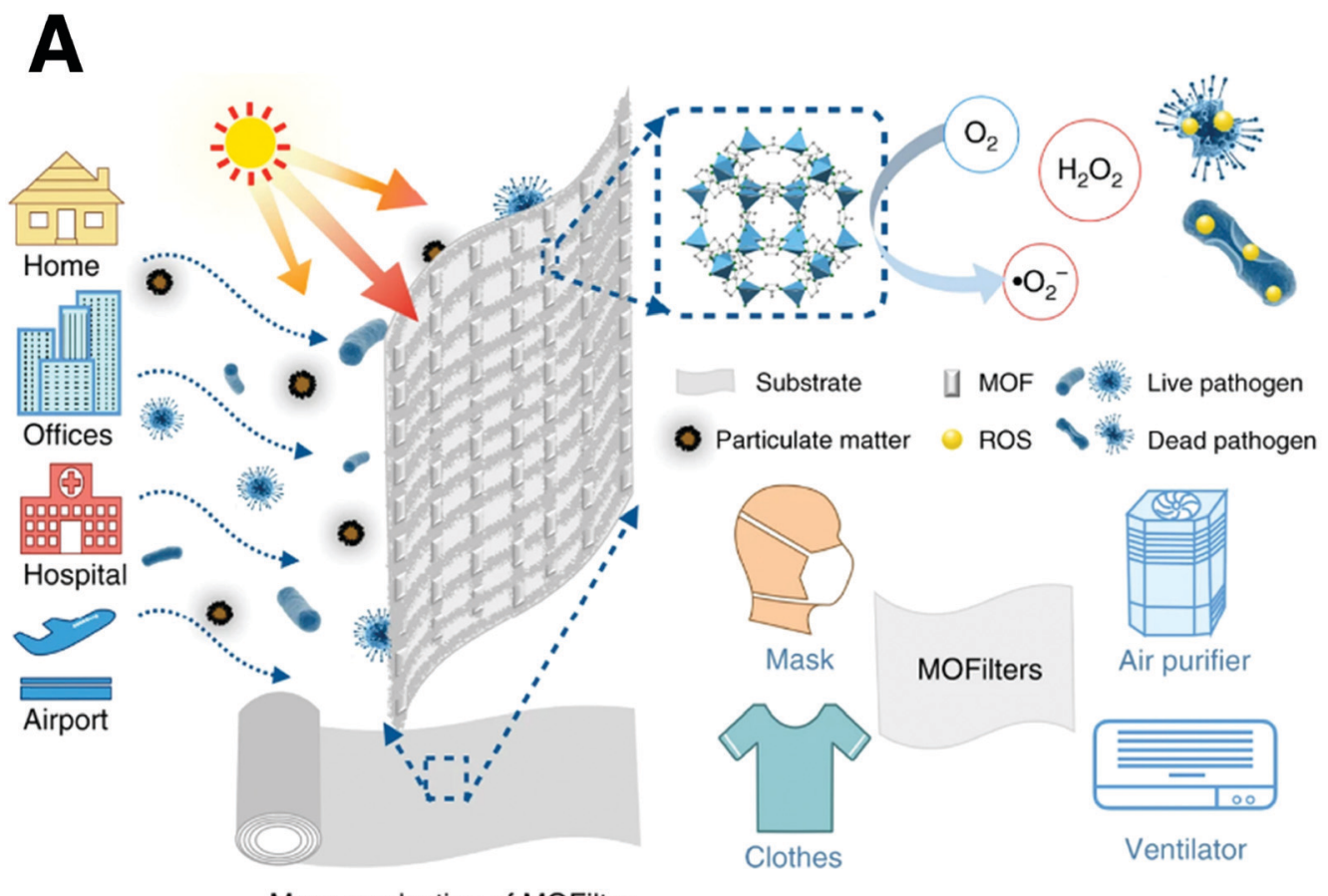

Mass production of MOFilter
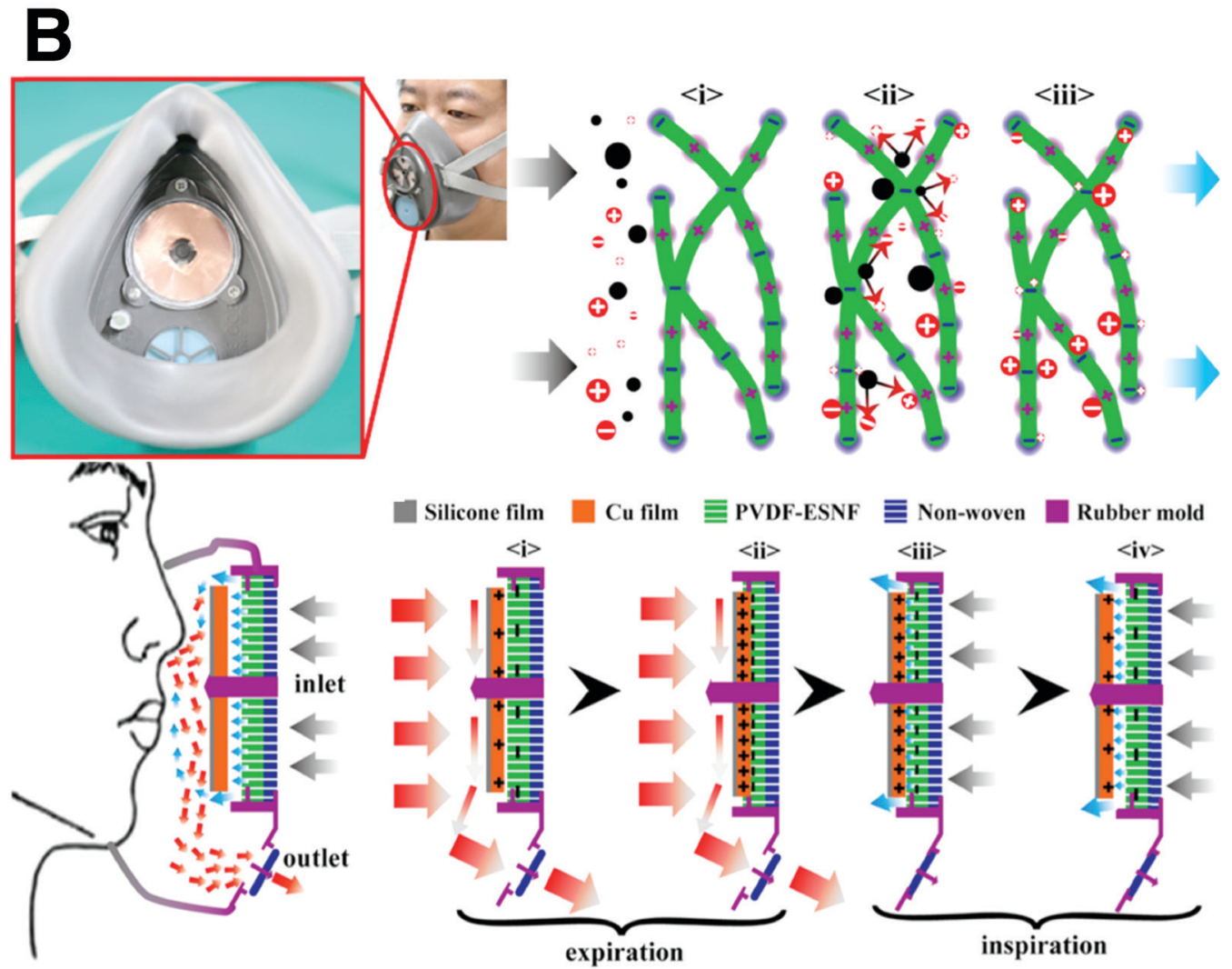

Fig. 10 Biosafety materials for PPE with antimicrobial capacity. (A) Schematic illustration of metal-organic framework (MOF)-based filter. Reproduced with permission from ref. 137. Copyright 2019, Nature. (B) Schematic illustration of the filtration mechanism of the polymeric material mask. Reproduced with permission from ref. 138. Copyright 2018, American Chemical Society.

animal and plant genetic materials such as biological genes, sperm and eggs. ${ }^{147-150}$ The major shortcomings for the current protection methods are the low survival rate of seeds and possibility of uncontrollable mutation. Cheng et al. discovered that PVP (polyvinylpyrrolidone) and PVPP (polyvinylpolypyrrolidone) could effectively remove multiple phenolic compounds and terpenoids 
during the process of extracting DNA. ${ }^{151}$ Therefore, the addition of an appropriate amount of PVP or PVPP to the DNA extraction solution can improve DNA purity, remove polysaccharides, and reduce polyphenol contamination. This is a good example that biosafety materials display protective significance on genetic resources. The PVP method has now been accepted as one of the most used DNA extraction techniques, thereby indicating that the development of materials actually changes the technic in protecting biological resouces. ${ }^{152-155}$

The protection of plant seeds is also a crucial biosafety issue. ${ }^{156,157}$ Seed enhancement technologies play a pivotal role in supporting food security by enabling the germination of seeds in degraded environments. Marelli et al. combined silkworm cocoon $\mathrm{S}$ molecules and trehalose to design a new seed coating method. ${ }^{158}$ As shown in Fig. 11A, this formulation is capable of precisely coating seeds with biofertilizers and releasing them in the soil to boost seed germination and mitigate soil salinity. The coated seeds yielded plants that grew faster and stronger in the presence of saline soil. Hence, this study opens the door to the application of advanced biosafety materials in precision agriculture, introducing the drug delivery concept to seed protection. Furthermore, polymeric materials have been proven to reduce environmental side effects and improve seed survival, suggesting the importance of composite materials in the protection of biological resources. ${ }^{159-162}$

Cryopreservation is the classical way to preserve human genetic resources. ${ }^{163}$ However, the mechanical damage effect of ice crystals is the major problem in cryopreservation. Inspired by the regenerable solid epicuticular wax on the surface of land plant leaves, Wang et al. developed solid organogel materials with a regenerable sacrificial alkane surface (Fig. 11B). ${ }^{164}$ This type of surface material was demonstrated to be of great
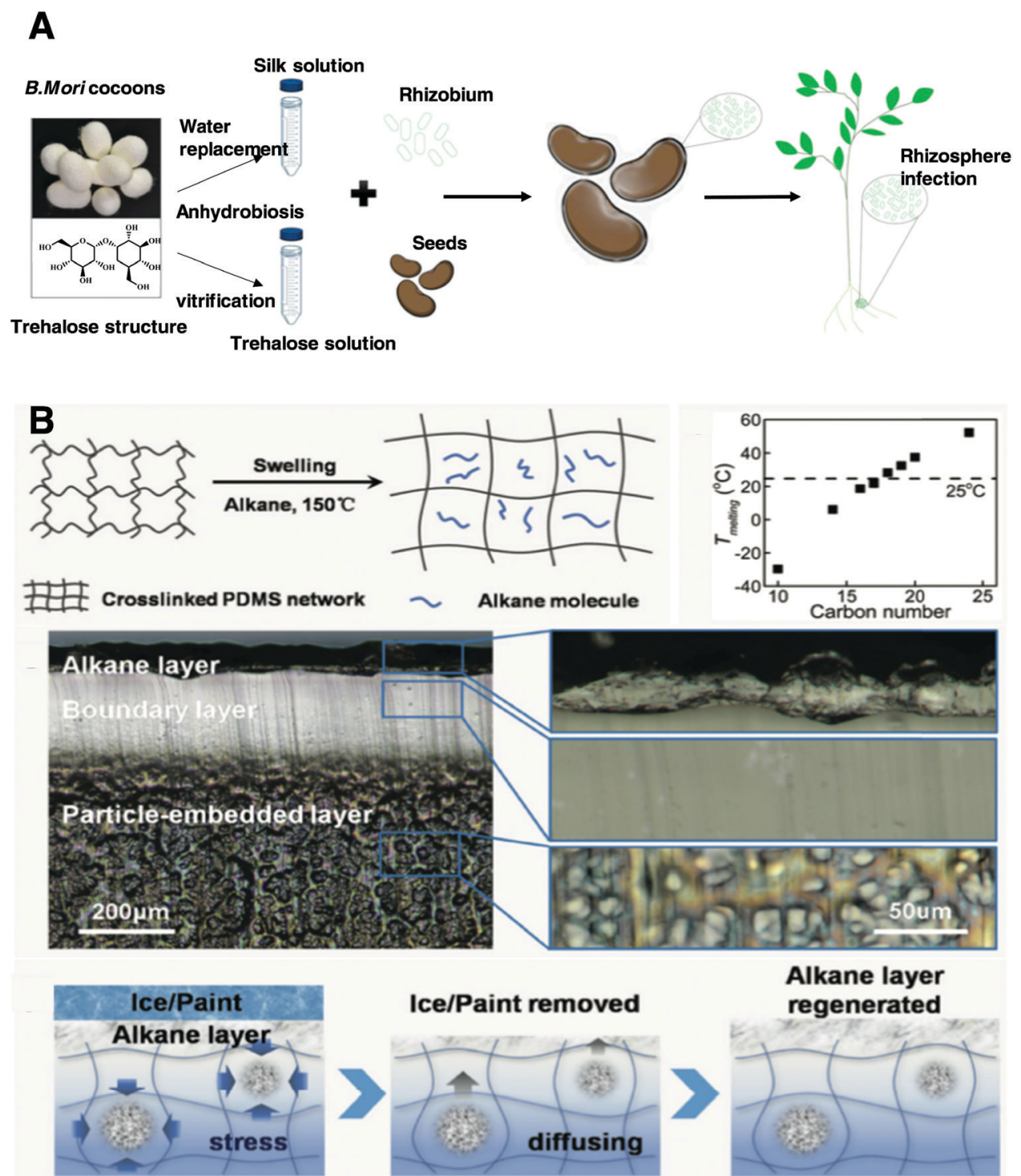

Fig. 11 Biosafety materials for the protection and preservation of biological resources. (A) Biosafety materials based on silk fibroin and trehalose for seed coating, which can boost germination and mitigate abiotic stress. Reproduced with permission from ref. 158. Copyright 2019, National Academy of Sciences. (B) Principle of the solid organogel material to cryopreserve biological resources. Reproduced with permission from ref. 164. Copyright 2017, Wiley-VCH. 
practical importance for tackling solid deposition, such as antiicing, anti-graffiti, and antifouling. Compared with the ice adhesion strength of aluminum $(372.2+47.4 \mathrm{kPa})$ and PDMS $(146.3+9.5 \mathrm{kPa})$, the ice adhesion strength of the solid organic gel was reduced to $68.8+10.4 \mathrm{kPa}$. The adhesion value remained almost unchanged when the temperature decreased from $-20{ }^{\circ} \mathrm{C}$ to $-70{ }^{\circ} \mathrm{C}$, indicating its potential for the cryopreservation of natural biological resources.

\subsection{Biosafety materials for the prevention of biological invasion}

Biological species invasion has been considered as one of the most critical ecological disturbances that threatens the native biodiversity. ${ }^{165-167}$ In 1996, the Global Invasive Species Program was launched. Since then, biological invasion has been a focus and heavily studied. ${ }^{168,169}$ Biological invasions can be divided into several stages, including introduction, escape, population establishment and harm. Except for purposeful introduction, the early detection of other invasion ways is challenging. If the invasion reaches a detectable range, it is hard to be removed. Hence, early detection is crucial to prevent biological invasion. The development of genetic detection technology has facilitated the detection of biological invasions. Wang et al. ${ }^{170}$ developed a sensitive nucleic-acid sensing platform based on superhydrophilic microwells spotted on a superhydrophobic substrate (Fig. 12). The difference in wettability facilitated the conversion of trace analyte between the micropores and surrounding substrate into superhydrophilic microporous materials. Due to the condensation enrichment effect, ultratrace DNA detection was realized, and the detection limit reached $2.3 \times 10^{-16} \mathrm{M}$. The genome of certain species can be rapidly determined using a gene library, enabling the fast identification of invading species.

Since invasive alien species have no natural enemy, they can rapidly settle in a new environment and quickly grow into an overlord community, eventually destroying the local ecosystem and inhibiting the growth of other local species. The loss of biodiversity in ecosystems further leads to irreversible consequences, such as the disappearance of forests, pastures degradation, and water pollution. Hence, in addition to the early detection of biological invasion, it is of great significance to effectively eliminate invaded alien species. Fortunately, the biosynthesis of metal nanoparticles provides an environmentally friendly approach to fight against invading alien species. Oscillatoria, as one of the most common cyanobacterial genera known to produce neurotoxins, has negative impacts on aquatic organisms. To stop the growth of Oscillatoria, the Ag-NPs biosynthesized by specific alga exert outstanding negative impacts on it, which significantly alleviates the environment burden through green synthesis. ${ }^{171}$ A similar biosynthesis strategy has also been found for controlling vector mosquitoes. The plant-synthesized AgNPs developed by Rajakumar et al. can effectively eliminate mosquitoes, providing an effective way to control mosquito-borne diseases. ${ }^{172}$ Hence, the development of materials science provides bio-friendly alternatives for the prevention of biological invasion, enriching the methods to deal with difficult environmental-related issues.

\subsection{Biosafety materials for protection of ecological environment}

The ecological environment is a prerequisite for ensuring species diversity. The 2010 Deepwater Horizon oil spill in the Gulf of Mexico has been regarded as the worst environmental disaster in the United States, which released about 4.9 million barrels of crude oil, and had a huge impact on the ecological system. Thus, efficient and cost-effective separation strategies for spilled oil are greatly needed. Janus particles are colloidal particles with more than one type of surface chemistry or composition, which provide a suitable system for oil separation. Song et al. developed magnetic Janus particles with a convex hydrophilic surface/concave oleophilic surface, realizing the rapid and efficient separation of microscale tiny oil droplets from water (Fig. 13). ${ }^{173}$ Ren et al. ${ }^{174}$ developed a phase-selective organic gelling agent, which not only showed the ability to selectively condense oil from oily water, but also could easily separate colloidal oil and water from the human body. This powder gelator exhibited remarkable ability to achieve rapid gelation of crude oils of widely ranging viscosities within minutes at room temperature.
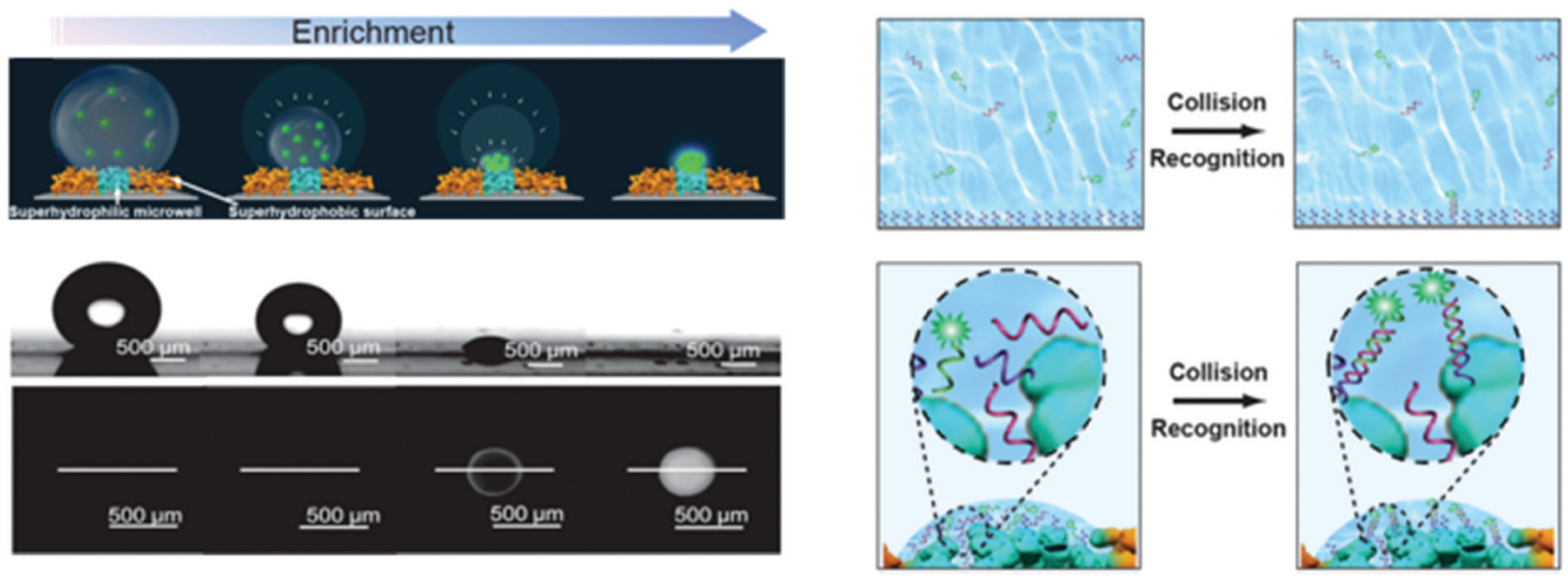

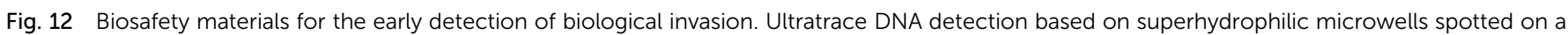
superhydrophobic substrate. Reproduced with permission from ref. 170. Copyright 2015, Wiley-VCH. 


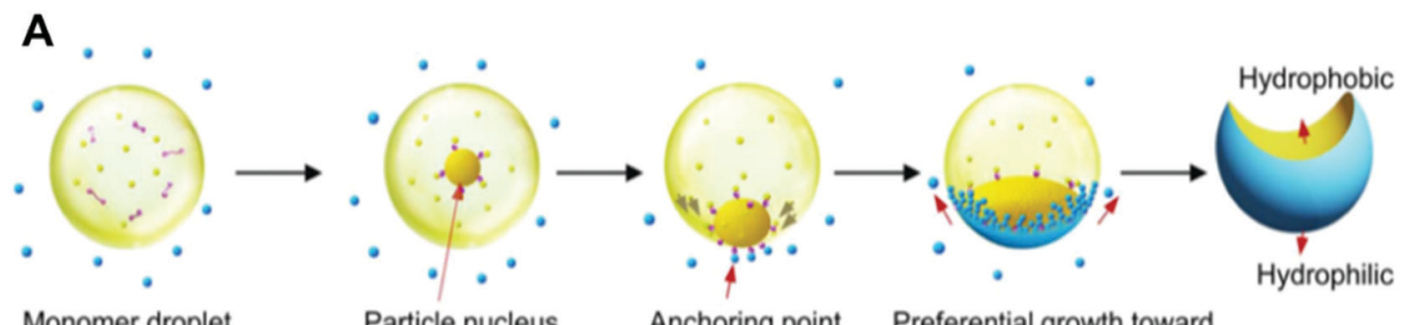

Monomer droplet Particle nucleus Anchoring point

two directions
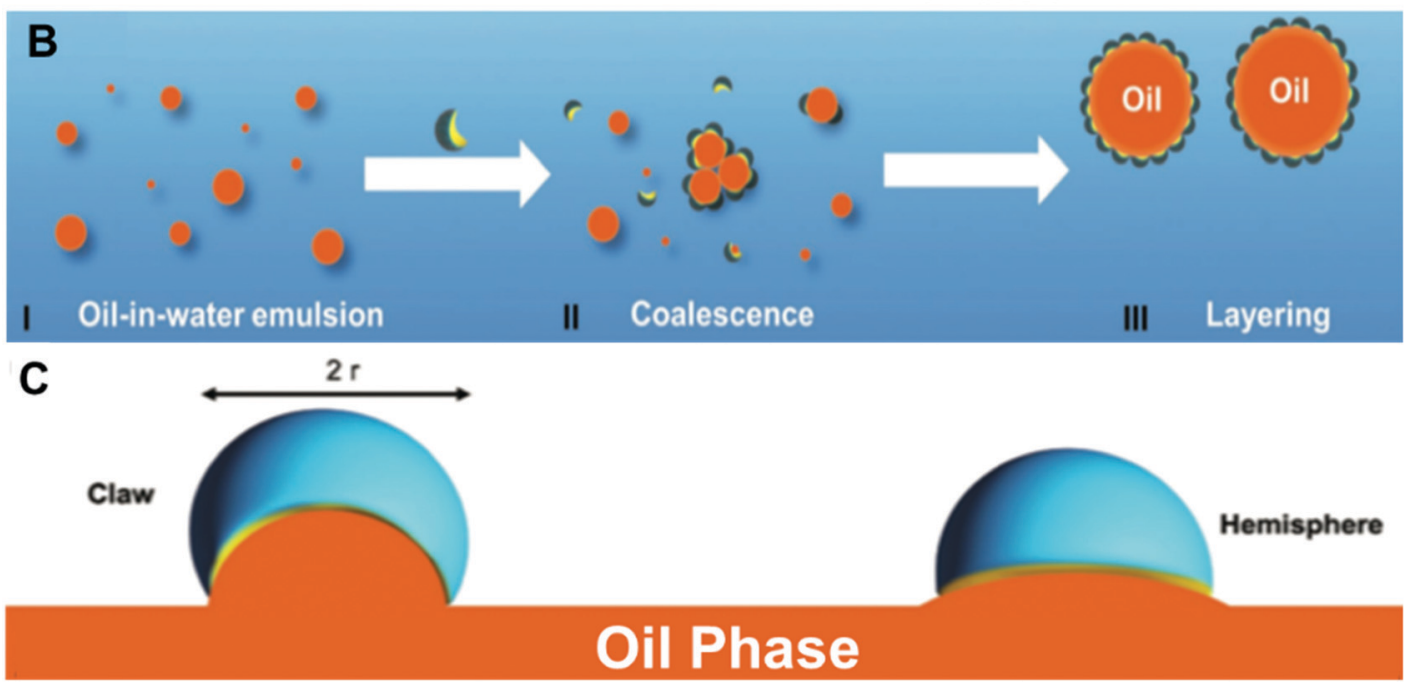

Fig. 13 Biosafety materials for separating spilled oil from water. (A) Schematic illustration of the principle of hydrophilic/oleophilic magnetic Janus particles. (B) Schematic of the separation of tiny oil droplets by Janus particles. (C) Schematic of two different types of Janus particles. Reproduced with permission from ref. 173. Copyright 2018, Wiley-VCH.

Heavy metals are harmful and toxic pollutants that are difficult to degrade. They not only cause a degradation in soil quality, but also enter the human body through direct contact or the food chain. In recent years, scientists have gradually realized that heavy metals have a massive impact on the microbes in soil, which eventually influence the soil microbial activity, microbial community, and soil enzyme activity. Thus, to ameliorate this condition, scientists have developed novel materials to remove heavy metal pollution in the soil ecosystem. ${ }^{175}$ Lian et al. developed a simple and economical path to prepare mercaptofunctionalized nanosilica. ${ }^{176}$ Nanosilica, which has excellent compatibility with soils, was chosen as the matrix. This biosafety material efficiently remediated $\mathrm{Pb} / \mathrm{Cd}$-contaminated soils, exhibiting a high immobilization efficiency of $99.12 \%$ and $98.23 \%$ towards $\mathrm{Pb}$ and Cd, respectively. ${ }^{176}$ In addition to silica, carbon-based materials have also been selected to deal with this problem due to their excellent absorption capacity, providing versatile methods to protect the ecological environment. ${ }^{177,178}$

\subsection{Biosafety materials for protection against bioterrorism}

Bioterrorist attacks have been regarded as the most important biosecurity threat in modern society. Monitoring and medical and health response are the two critical measures for preventing bioterrorism. The biological warfare agents that terrorists can use for attack include Bacillus anthracis, Brucella, Rickettsia przewalskii, and Yersinia pestis. These agents are mainly spread through aerosols, food and water sources. Bioterrorism possesses the characteristics of strong infectivity, strong concealment, simple production process, and wide impact. Anthrax spores are an ideal biological weapon since they are highly lethal to human beings and animals. ${ }^{179-181}$ Thus, rapid detection methods for anthrax spores are in great demand. As shown in Fig. 14, Tang et al. designed a rare earth-functionalized micelle nanoprobe for the ratiometric fluorescence detection of an anthrax spore biomarker, pyridine dicarboxylic acid (DPA). ${ }^{182}$ The detection strategy was ascribed to the $\mathrm{Tb}^{3+}$ ions in the lanthanide-functionalized micelle, which can be sensitized to emit intrinsic luminescence upon the addition of DPA due to the occurrence of energy transfer when the DPA chromophore coordinates with the $\mathrm{Tb}^{3+}$ ion. This nanoprobe can detect DPA within a linear range of $0-7 \mu \mathrm{M}$ within a few seconds, and the detection limit is up to $54 \mathrm{nM}$. It is believed that the defense against biological weapons can be strengthened by incorporating novel materials with peculiar properties into the detection system.

Because of the acute neurotoxicity caused by biological weapons, saving victims after exposure remains challenging. Neurodrugs are organophosphorus compounds (OPS) that block the communication between the nerves and organs, which have been used as biological weapons. ${ }^{183-185}$ Jiang et al. developed a nanoscavenger, which exhibited a long-term protective effect on OPS poisoning in rodents (Fig. 15) ${ }^{186}$ It could catalyze the decomposition of toxic OPS, and exhibited excellent pharmacokinetic 


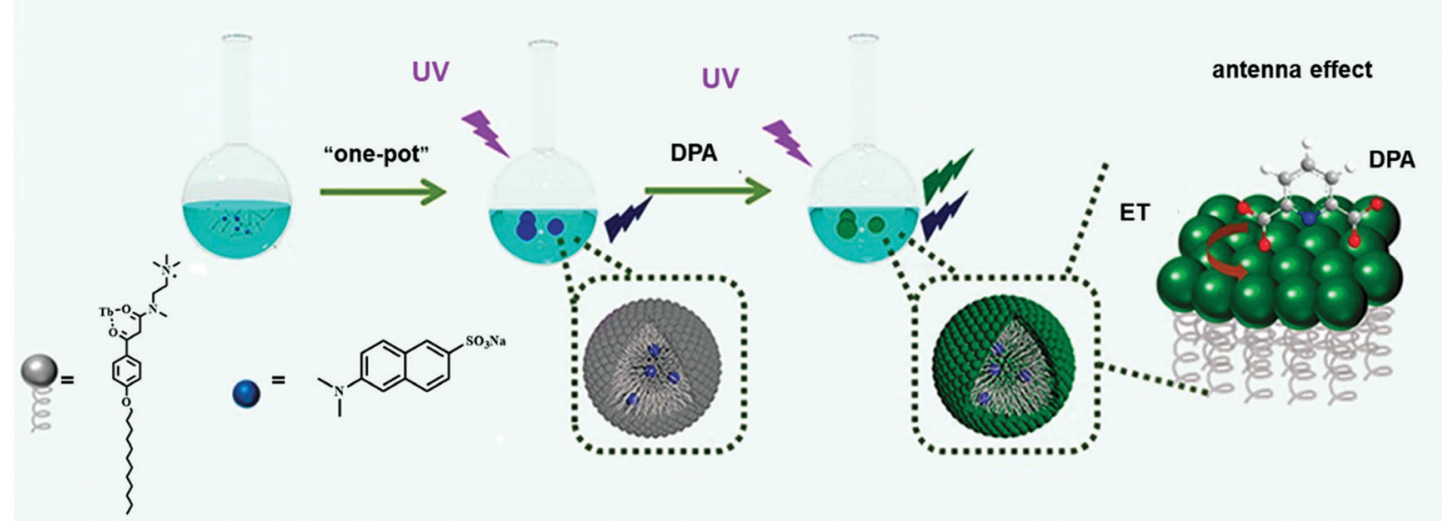

Fig. 14 Biosafety materials based on a ratiometric fluorescence lanthanide-functionalized micelle for the detection of an anthrax spore biomarker. Schematic illustration of the "one-pot" self-assembly of terbium-functionalized micelle in $\mathrm{H}_{2} \mathrm{O}$ and its response property for DPA. Reproduced with permission from ref. 182. Copyright 2018, American Chemical Society.

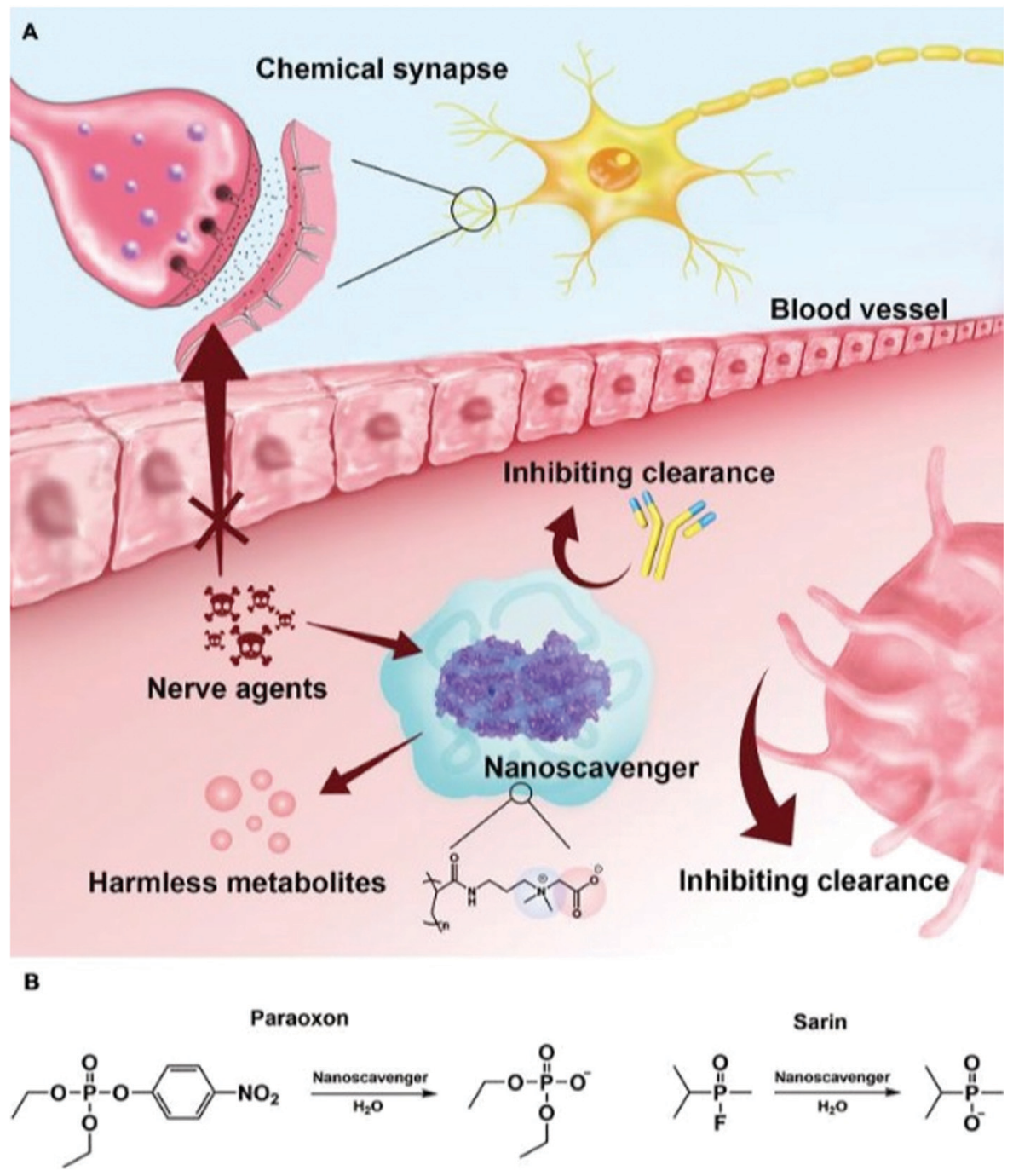

Fig. 15 Biosafety materials as nanoscavengers to remove neuro-drugs. (A) Schematic illustration of the protection mechanism of nanoscavengers. (B) Hydrolysis of paraoxon and sarin mediated by nanoscavengers. Reproduced with permission from ref. 186. Copyright 2019, American Association for the Advancement of Science. 
characteristics and negligible immune response in OP-poisoned rats. In a guinea pig model, the single prophylactic administration of nanoscavengers effectively prevented death after repeated exposure to sarin within one week, demonstrating the translational significance of nanoscavengers in clinical and military settings.

\subsection{Biosafety materials for genetic technology}

The emergence of genetic technology directly broke the original pattern of science and promoted the development of medical, agricultural and other fields. However, it also brought problems such as gene mutation and hybridization, indicating the double-sidedness of gene technology. The conventional genetic engineering technique targets the nuclear genome, resulting in problems in the proliferation of foreign genes to weedy relatives. Target delivery to specific organelles is highly desirable for plant genetic engineering, which can be achieved via nanoparticlemediated transformation. Wong et al. designed a gene carrier based on chitosan-complexed single-walled carbon nanotubes (SWNTs) for chloroplast transformation. ${ }^{187}$ The nanotube carrier could deliver plasmid DNA to the chloroplast of different plant species without external biolistic or chemical aid, thereby rendering a chloroplast transgene delivery platform for mature plants across different species. In Fig. 16, Wang et al. summarized a series of functionalized nanomaterials that provide diverse platforms capable of traversing barriers (e.g., multilayered cell walls) to deliver exogenous plasmid pDNA and siRNA in to intact plant cells. ${ }^{188}$

\subsection{Biosafety materials for food safety}

Food safety, as a global public health issue, has been regarded as a biosafety problem. ${ }^{189-191}$ The major issues in food safety are summarized as follows. (1) The presence of microorganisms directly causes mold, which can easily spread during the process of production, distribution and packaging. ${ }^{192-195}$ (2) The emergence of additives greatly helps in the manufacture of food that meets certain requirements, but if the amount of food additives used exceeds the standard level, it poses a serious threat to human health. ${ }^{196,197}$ (3) The overuse of chemical fertilizers and pesticides leads to severe pollution and food safety problems, which places a vast burden on human health. ${ }^{198,199}$ Thus, to tackle these challenges, materials scientists have proposed various solutions for food safety problems in testing, packaging, and storage. ${ }^{200-203}$

Hydrogen sulfide $\left(\mathrm{H}_{2} \mathrm{~S}\right)$ with rotten egg odor is produced in rotten food. Thus, the rapid and sensitive detection of $\mathrm{H}_{2} \mathrm{~S}$ is important to forewarn of food spoilage or pollution incidents with respect to this gas. Tang et al. prepared an Ag@Au coreshell nanoprobe combined with headspace single-drop microextraction (HS-SDME). ${ }^{204}$ Smartphone nanocolorimetry with the aid of a smartphone camera and color picker software was applied to detect and quantify $\mathrm{H}_{2} \mathrm{~S}$ (Fig. 17). The limit of detection for this nanocolorimetric approach was as low as $65 \mathrm{nM}$, which represents an ideal in situ analytical approach for the determination of $\mathrm{H}_{2} \mathrm{~S}$. Hence, the development of biosafety materials provides technical support for food safety.

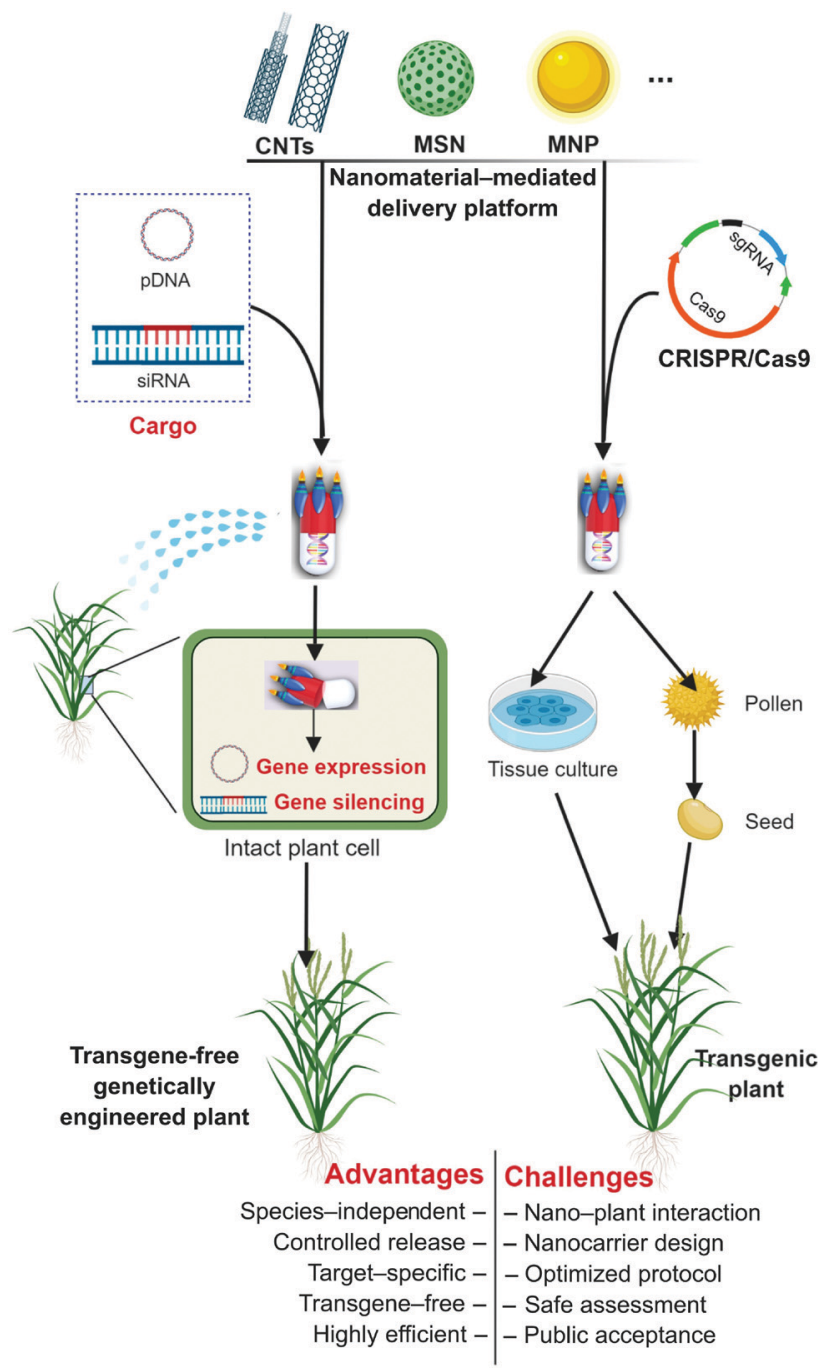

Fig. 16 Biosafety materials for plant genetic engineering. Multiple nanotechnology-based materials and cargos for gene delivery have been developed, improving our ability to specifically deliver gene-editing agents. Reproduced with permission from ref. 188. Copyright 2019, Elsevier.

\subsection{Biosafety materials for aerospace safety}

The space radiation environment is mainly composed of Milky Way cosmic rays, solar high-energy particles, and particles in the radiation zone of the near-Earth anomaly zone. After longterm analysis of the space radiation environment, NASA officially listed astronauts as radioactive workers in the 1980s, which indicates that aerospace safety is a biosafety problem. Doherty et al. reported a high-absorptivity/high-emissivity bone charbased thermal control surface known as SolarBlack for use on rigid and flexible metallic substrates, including titanium, aluminium, copper alloys (Fig. 18). ${ }^{205}$ This technology has been qualified for use on the front surface of the Solar Orbiter heat shield, thereby providing astronauts with biological safety protection. ${ }^{206}$

The harm caused by an impact accident in a space environment can directly lead to a devastating consequence. Thus, a polymer material that can self-heal within one second after impact has been developed to solve this problem. ${ }^{207}$ The rapid 


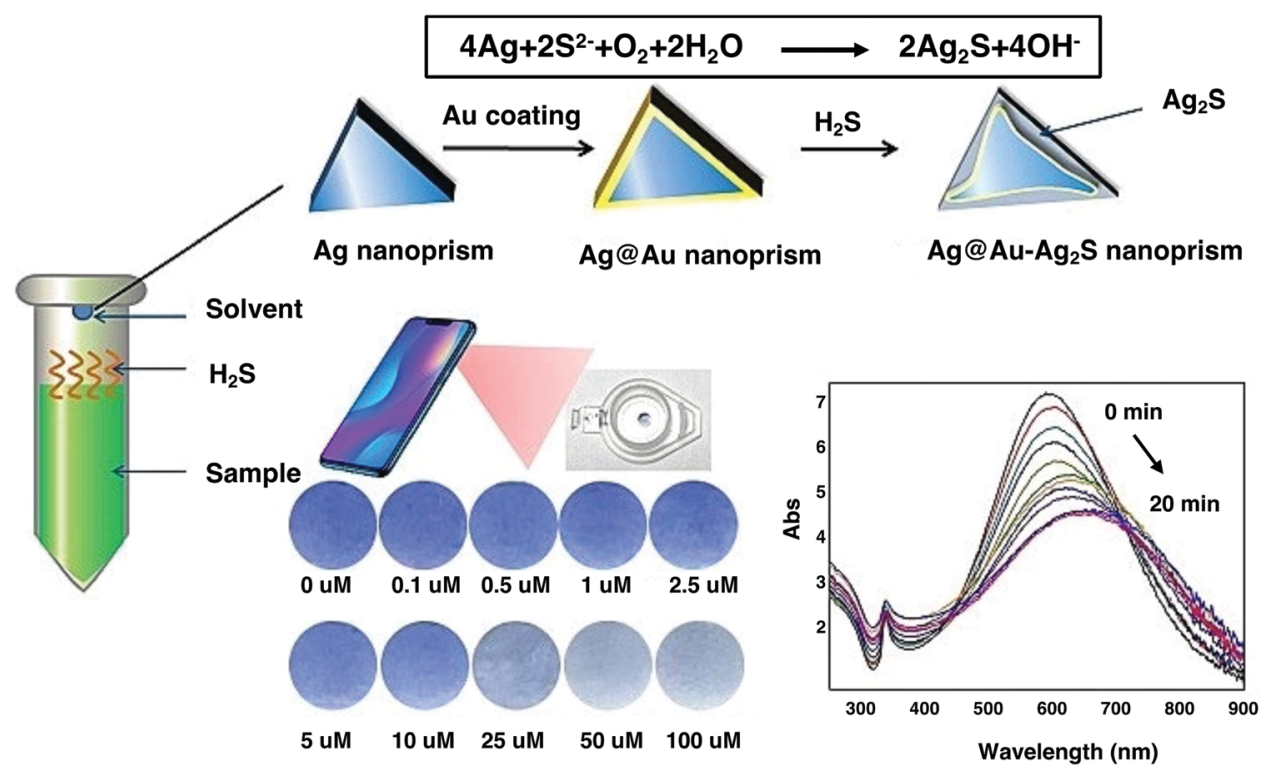

Fig. 17 Biosafety materials for food safety. Schematic illustration of the $\mathrm{H}_{2} \mathrm{~S}$ sensing mechanism based on the Ag@Au core-shell nanoprobe. Reproduced with permission from ref. 204. Copyright 2019, American Chemical Society.
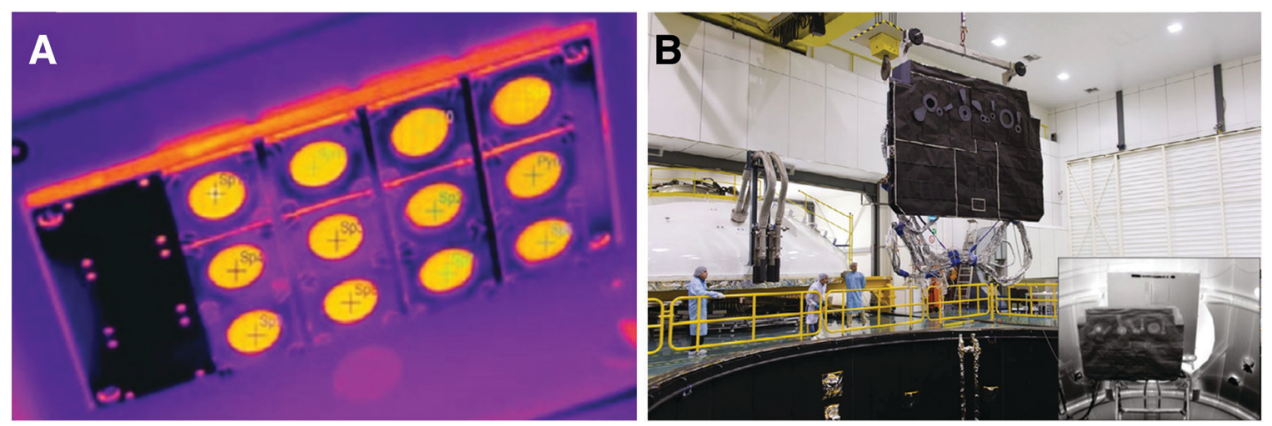

Fig. 18 Biosafety materials for aerospace safety. (A) SolarBlack material samples during testing in the Synergist Temperature Accelerated Testing (STAR) facility in the ESA/ESTEC center. (B) Solar orbiter heatshield based on SolarBlack technology. Reproduced with permission from ref. 205. Copyright 2015, Elsevier.

reaction rates was achieved by thiol-ene-alkylborane formulations, providing astronauts with biological safety protection at the physical level, which ensures the safety of human life and improves the efficiency of related work in the process of exploring space.

\section{Outlook and prospects}

In summary, a variety of materials, such as polymers, MOFs, graphene, and carbon nanotubes, have dramatically changed our strategy in facing grand challenges for biosafety, but no clear definition and detailed plan on the development of biosafety materials have been given to date. Numerous reports on the progress of materials science prove its capacity to solve the difficult biosafety problems. To the best of our knowledge, we are the first to officially propose the brand-new concept of "biosafety materials" to specify the role of materials science in biosafety, aiming at raising the awareness of the scientific community to actively integrate the two different subjects and advocate the marriage of both concepts. This brand-new concept of "biosafety materials" can help solve problems related to biosafety. Since biosafety covers a wide range of topics including pathogen detection, biological invasion prevention, protection and prevention of biological resources, and genetic technology, solely relying on a single discipline to address these issues in biosafety is impossible. Thus, the integration of biosafety and materials science can significantly facilitate the development of effective biosafety materials, thus providing a powerful toolbox for professionals to solve biosafety-related problems.

Overall, despite the significant progress in recent years, the development of biosafety materials is still in the early stage, and great efforts should be made to improve the current technologies and facilitate the development of biosafety materials. Thus, the following measures are suggested.

Firstly, biosafety materials science majors should be opened in universities and research institutes to strengthen biosafety materials science disciplines, train professional teams, and direct biosafety materials professionals in basic research in the field of 
infectious diseases. The development of biosafety materials can support future work on the traceability and transmission of pathogens of high incidence, sudden infectious diseases, understanding infection and pathogenic mechanisms, and determining anti-infection means. Secondly, scientific research laboratories and research centers related to biosafety materials science and research platforms should be built to strengthen the integration of relevant scientific research, which can finally improve the biosafety research system. Thirdly, professional associations related to biosafety materials science should be established, and developing professional journals and magazines to expand the influence of biosafety materials science is necessary. Finally, well-known enterprises specialized in biosafety materials to develop related products and equipment for biosafety issues should be formed. Biosafety materials research and development centers could be further built in these enterprises to support human health, social stability, and national security.

Taken together, globalization makes the current biosafety threat not only a problem for a single country. No country can protect itself from biological risks without cooperation with other countries. Hence, we hope scientists worldwide will share data and information to use biosafety materials collaboratively to tackle biosafety risks. Finally, we sincerely hope that biosafety materials science will become an independent discipline in the near future, and can flourish worldwide. The government will soon pay more attention to the development of biosafety materials science. An increasing number of researchers can realize its importance and join the research community to explore more and more biosafety materials as well as related products and equipment. Ultimately, the development of biosafety materials science will provide a solid guarantee for the health and wellbeing of society, economic prosperity, and national security worldwide.

\section{Conflicts of interest}

There are no conflicts to declare.

\section{Acknowledgements}

This work was supported by National Natural Science Foundation of China (51873218).

\section{Notes and references}

1 J. Y. Richmond, R. H. Hill, R. S. Weyant, S. L. Nesby-O'Dell and P. E. Vinson, What's hot in animal biosafety?, ILAR J., 2003, 44, 20-27.

2 M. U. Kraemer, C.-H. Yang, B. Gutierrez, C.-H. Wu, B. Klein, D. M. Pigott, L. du Plessis, N. R. Faria, R. Li and W. P. Hanage, The effect of human mobility and control measures on the COVID-19 epidemic in China, Science, 2020, 368, 493-497.

3 J. Thornton, Don't forget chronic lung and immune conditions during covid-19, says WHO, BMJ, 2020, 368, m1192.
4 T. Wang, Z. Du, F. Zhu, Z. Cao, Y. An, Y. Gao and B. Jiang, Comorbidities and multi-organ injuries in the treatment of COVID-19, Lancet, 2020, 395, e52.

5 X. Wang, COVID-19 Epidemic and Enhancing China's National Biosecurity System, J. Biosafety Biosecur., 2020, DOI: 10.1016/j.jobb.2020.03.002.

6 M. B. Forrester and S. K. Stanley, Calls about anthrax to the Texas Poison Center Network in relation to the anthrax bioterrorism attack in 2001, Vet. Hum. Toxicol., 2003, 45, 247-248.

7 W. H. Organization, Laboratory biosafety guidance related to coronavirus disease 2019 (COVID-19): interim guidance, 12 February 2020, World Health Organization, 2020.

8 O. Akan, Laboratory infections, Mikrobiyol. Bul., 1993, 27, 77-84.

9 S. F. Lin, P. L. Jiang, J. S. Tsai, Y. Y. Huang, S. Y. Lin, J. H. Lin and D. Z. Liu, Surface assembly of poly (I: C) on polyethyleneimine-modified gelatin nanoparticles as immunostimulatory carriers for mucosal antigen delivery, J. Biomed. Mater. Res., Part B, 2019, 107, 1228-1237.

10 M. Lian, B. Sun, Z. Qiao, K. Zhao, X. Zhou, Q. Zhang, D. Zou, C. He and X. Zhang, Bi-layered electrospun nanofibrous membrane with osteogenic and antibacterial properties for guided bone regeneration, Colloids Surf., B, 2019, 176, 219-229.

11 C. Bachireddy, C. Chen and M. Dar, Securing the Safety Net and Protecting Public Health During a Pandemic: Medicaid's Response to COVID-19, J. Am. Med. Assoc., 2020, DOI: 10.1001/jama.2020.4272.

12 T. Trevan, Biological research: rethink biosafety, Nat. News, 2015, 527, 155.

13 D. P. Fidler, Medicine at the Border, Springer, 2007, pp. 196-218.

14 M. Liapin and V. Kutyrev, Actual problems of biosafety, $Z$. Mikrobiol., Epidemiol. Immunobiol., 2013, 97-102.

15 C. L. Briggs, Communicating biosecurity, Med. Anthropol, 2011, 30, 6-29.

16 G. Borkow, S. S. Zhou, T. Page and J. Gabbay, A novel antiinfluenza copper oxide containing respiratory face mask, PLoS One, 2010, 5(6), e11295.

17 L. Bakanidze, P. Imnadze and D. Perkins, Biosafety and biosecurity as essential pillars of international health security and cross-cutting elements of biological nonproliferation, BMC Public Health, 2010, 10, S12.

18 L. A. Meyerson and J. K. Reaser, Biosecurity: Moving toward a comprehensive approach, Bioscience, 2002, 52, 593-600.

19 S. E. Davies, Biosecurity interventions: global health and security in question, Int. Aff., 2009, 85, 639.

20 C. Humphreys, Materials science and engineering in Britain, Angew. Chem., Int. Ed. Engl., 1989, 28, 1077-1078.

21 T. N. Nguyen, T. N. Huynh, D. Hoang, D. H. Nguyen, Q. H. Nguyen and T. H. Tran, Functional Nanostructured Oligochitosan-Silica/Carboxymethyl Cellulose Hybrid Materials: Synthesis and Investigation of Their Antifungal Abilities, Polymers, 2019, 11, 628.

22 R. J. Nevagi, M. Skwarczynski and I. Toth, Polymers for subunit vaccine delivery, Eur. Polym. J., 2019, 114, 397-410. 
23 M. Nautiyal, S. De Graef, L. Pang, B. Gadakh, S. V. Strelkov, S. D. Weeks and A. Van Aerschot, Comparative analysis of pyrimidine substituted aminoacyl-sulfamoyl nucleosides as potential inhibitors targeting class I aminoacyl-tRNA synthetases, European, J. Med. Chem., 2019, 173, 154-166.

24 J. Li, Y. Yu, K. Myungwoong, K. Li, J. Mikhail, L. Zhang, C.-C. Chang, D. Gersappe, M. Simon and C. Ober, Manipulation of cell adhesion and dynamics using RGD functionalized polymers, J. Mater. Chem. B, 2017, 5, 6307-6316.

25 F. Eggiman, U. Meier and H. W. Fritz, EMPA and its activities in the field of building materials, elements and structures, Mater. Struct., 1995, 28, 101-102.

26 S. Feng, C. Shen, N. Xia, W. Song, M. Fan and B. J. Cowling, Rational use of face masks in the COVID-19 pandemic, Lancet Respir. Med., 2020, 8, 434-436.

27 E. Mahase, Covid-19: UK could delay non-urgent care and call doctors back from leave and retirement, BMJ, 2020, 368, m854.

28 E. Livingston, A. Desai and M. Berkwits, Sourcing personal protective equipment during the COVID-19 pandemic, J. Am. Med. Assoc., 2020, 323, 1912-1914.

29 M. A. Matthay, J. M. Aldrich and J. E. Gotts, Treatment for severe acute respiratory distress syndrome from COVID-19, Lancet Respir. Med., 2020, 8, 433-434.

30 E. Mahase, Coronavirus: home testing pilot launched in London to cut hospital visits and ambulance use, $B M J$, 2020, 368, m621.

31 N. Greenberg, M. Docherty, S. Gnanapragasam and S. Wessely, Managing mental health challenges faced by healthcare workers during covid-19 pandemic, BMJ, 2020, 368, m1211.

32 J. Ma, X. Cheng, F. Peng, N. Zhang, R. Li, L. Sun, Z.-L. Li and $\mathrm{H}$. Jiang, A polymer dots fluorescent sensor for detection of alkaline phosphatase activity and inhibitor evaluation, J. Mater. Sci., 2019, 54, 10055-10064.

33 H. S. Maghdid, K. Z. Ghafoor, A. S. Sadiq, K. Curran and K. Rabie, A novel ai-enabled framework to diagnose coronavirus covid 19 using smartphone embedded sensors: Design study, 2020, arXiv preprint arXiv:2003.07434.

34 L. Wang, Z. Yuan, H. E. Karahan, Y. Wang, X. Sui, F. Liu and Y. Chen, Nanocarbon materials in water disinfection: state-of-the-art and future directions, Nanoscale, 2019, 11, 9819-9839.

35 W.-F. Lai and A. L. Rogach, Hydrogel-based materials for delivery of herbal medicines, ACS Appl. Mater. Interfaces, 2017, 9, 11309-11320.

36 D. Irvine, Material aid for vaccines, Nat. Mater., 2018, 17, 472-473.

37 S. P. Levine, M. A. Puskar, C. L. Geraci, A. A. Grote and M. Bolyard, Fourier Transform Infra-Red Spectroscopy Applied to Hazardous Waste: I-Preliminary Test of Material Analysis for Improvement of Personal Protection Strategies, Am. Ind. Hyg. Assoc. J., 1985, 46, 181-186.

38 M. Hussain, J. Wackerlig and P. A. Lieberzeit, Biomimetic strategies for sensing biological species, Biosensors, 2013, 3, 89-107.
39 D. A. Stringfellow, M. D. Givens and J. G. Waldrop, Biosecurity issues associated with current and emerging embryo technologies, Reprod., Fertil. Dev., 2003, 16, 93-102.

40 C. L. Hewitt, Marine biosecurity issues in the world oceans: global activities and Australian directions, Ocean Yearb. Online, 2003, 17, 193-212.

41 B. D. Nordmann, Issues in biosecurity and biosafety, Int. J. Antimicrob. Agents, 2010, 36, S66-S69.

42 H. Mukai and T. Nakagawa, Long and accurate PCR (LA PCR), Nihon rinsho. Jpn. J. Clin. Med., 1996, 54, 917-922.

43 I. Y. Yoo, J.-Y. Kim, Y. K. Yoon, H. J. Huh and N. Y. Lee, Comparison Between the SFTS-QS Kit and the PowerChek SFTSV Real-time PCR Kit for the Detection of Severe Fever With Thrombocytopenia Syndrome Virus, Ann. Lab. Med., 2020, 40, 317-320.

$44 \mathrm{H}$. Hyodo and H. Furuta, POCT and system, Rinsho Byori. Jpn. J. Clin. Pathol., 2002, 50, 940-946.

45 X. Huang, J. Li, M. Lu, W. Zhang, Z. Xu, B.-Y. Yu and J. Tian, Point-of-Care Testing of MicroRNA based on Personal Glucose Meter and Dual Signal Amplification to Evaluate DrugInduced Kidney Injury, Anal. Chim. Acta, 2020, 1112, 72-79.

46 H. Zhang, Z. Wang, Q. Zhang, F. Wang and Y. Liu, $\mathrm{Ti}_{3} \mathrm{C}_{2}$ MXenes nanosheets catalyzed highly efficient electrogenerated chemiluminescence biosensor for the detection of exosomes, Biosens. Bioelectron., 2019, 124, 184-190.

47 Q. Sui, P. Li, N.-N. Yang, T. Gong, R. Bu and E.-Q. Gao, Differentiable Detection of Volatile Amines with a Viologen-Derived Metal-Organic Material, ACS Appl. Mater. Interfaces, 2018, 10, 11056-11062.

48 J. H. Kim, J. E. Park, M. Lin, S. Kim, G. H. Kim, S. Park, G. Ko and J. M. Nam, Sensitive, Quantitative Naked-Eye Biodetection with Polyhedral Cu Nanoshells, Adv. Mater., 2017, 29, 1702945.

49 C. Vepari and D. L. Kaplan, Silk as a biomaterial, Prog. Polym. Sci., 2007, 32, 991-1007.

50 T. Chandy and C. P. Sharma, Chitosan-as a biomaterial, Biomater., Artif. Cells Artif. Organs, 1990, 18, 1-24.

51 K. J. Burg, S. Porter and J. F. Kellam, Biomaterial developments for bone tissue engineering, Biomaterials, 2000, 21, 2347-2359.

52 A. Subramanian, U. M. Krishnan and S. Sethuraman, Development of biomaterial scaffold for nerve tissue engineering: Biomaterial mediated neural regeneration, J. Biomed. Sci., 2009, 16, 108.

53 R. Rai, T. Keshavarz, J. Roether, A. R. Boccaccini and I. Roy, Medium chain length polyhydroxyalkanoates, promising new biomedical materials for the future, Mater. Sci. Eng., $R$, 2011, 72, 29-47.

$54 \mathrm{~J}$. Jones and L. Hench, Biomedical materials for new millennium: perspective on the future, Mater. Sci. Technol., 2001, 17, 891-900.

55 F. H. Silver and D. L. Christiansen, Biomaterials science and biocompatibility, Springer, 1999, pp. 1-26.

56 L. L. Hench and J. M. Polak, Third-generation biomedical materials, Science, 2002, 295, 1014-1017.

57 N. Nimi, A. Saraswathy, S. S. Nazeer, N. Francis, S. J. Shenoy and R. S. Jayasree, Biosafety of citrate coated 
zerovalent iron nanoparticles for Magnetic Resonance Angiography, Data Brief, 2018, 20, 1829-1835.

58 X. Huang, Y. Zhang, M. Shi, Y. Zhang and Y. Zhao, Study on a polymerizable visible light initiator for fabrication of biosafety materials, Polym. Chem., 2019, 10, 2273-2281.

59 Y. Sun, W. Feng, P. Yang, C. Huang and F. Li, The biosafety of lanthanide upconversion nanomaterials, Chem. Soc. Rev., 2015, 44, 1509-1525.

60 Y. Zhao, G. Xing and Z. Chai, Nanotoxicology: Are carbon nanotubes safe?, Nat. Nanotechnol., 2008, 3, 191.

61 L. Zhu, L. Wang, X. Zhang, T. Li, Y. Wang, M. A. Riaz, X. Sui, Z. Yuan and Y. Chen, Interfacial engineering of graphenic carbon electrodes by antimicrobial polyhexamethylene guanidine hydrochloride for ultrasensitive bacterial detection, Carbon, 2020, 159, 185-194.

62 J. Shi, M. Wang, Z. Sun, Y. Liu, J. Guo, H. Mao and F. Yan, Aggregation-induced emission-based ionic liquids for bacterial killing, imaging, cell labeling, and bacterial detection in blood cells, Acta Biomater., 2019, 97, 247-259.

63 Q. Li, Y. Wu, H. Lu, X. Wu, S. Chen, N. Song, Y.-W. Yang and $\mathrm{H}$. Gao, Construction of supramolecular nanoassembly for responsive bacterial elimination and effective bacterial detection, ACS Appl. Mater. Interfaces, 2017, 9, 10180-10189.

64 M. S. Draz and H. Shafiee, Applications of gold nanoparticles in virus detection, Theranostics, 2018, 8, 1985.

65 B. Yang, J. Kong and X. Fang, Bandage-like wearable flexible microfluidic recombinase polymerase amplification sensor for the rapid visual detection of nucleic acids, Talanta, 2019, 204, 685-692.

66 K. Liu, D. Pan, Y. Wen, H. Zhang, J. Chao, L. Wang, S. Song, C. Fan and Y. Shi, Identifying the genotypes of hepatitis B virus (HBV) with DNA origami label, Small, 2018, 14, 1701718.

67 Y. Yu, Q. Zhang, C.-C. Chang, Y. Liu, Z. Yang, Y. Guo, Y. Wang, D. K. Galanakis, K. Levon and M. Rafailovich, Design of a molecular imprinting biosensor with multiscale roughness for detection across a broad spectrum of biomolecules, Analyst, 2016, 141, 5607-5617.

68 V. Ricotta, Y. Yu, N. Clayton, Y.-C. Chuang, Y. Wang, S. Mueller, K. Levon, M. Simon and M. Rafailovich, A chip-based potentiometric sensor for a Zika virus diagnostic using 3D surface molecular imprinting, Analyst, 2019, 144, 4266-4280.

69 Y. T. Lim, Vaccine adjuvant materials for cancer immunotherapy and control of infectious disease, Clin. Exp. Vaccine Res., 2015, 4, 54-58.

70 K. Blecher, A. Nasir and A. Friedman, The growing role of nanotechnology in combating infectious disease, Virulence, 2011, 2, 395-401.

71 L. Rao, R. Tian and X. Chen, Cell-Membrane-Mimicking Nanodecoys against Infectious Diseases, ACS Nano, 2020, 14, 2569-2574.

72 Z. Liu, L. Zhang, Q. Guan, Y. Guo, J. Lou, D. Lei, S. Wang, S. Chen, L. Sun and H. Xuan, Biomimetic Materials with Multiple Protective Functionalities, Adv. Funct. Mater., 2019, 29, 1901058.
73 G. Gralewicz and B. Więcek, Active thermography in qualitative evaluation of protective materials, Int. J. Occup. Saf. Ergon., 2009, 15, 363-371.

74 N. Shimasaki, K. Shinohara and H. Morikawa, Performance of materials used for biological personal protective equipment against blood splash penetration, Ind. Health, 2017, 55, 521-528.

75 Y. Zhang, X. Le, Y. Jian, W. Lu, J. Zhang and T. Chen, 3D Fluorescent Hydrogel Origami for Multistage Data Security Protection, Adv. Funct. Mater., 2019, 29, 1905514.

76 P. F. Campos and T. M. Gilbert, Ancient DNA, Springer, 2012, pp. 81-85.

77 M. Pasha, C. Hare, M. Ghadiri, A. Gunadi and P. M. Piccione, Inter-particle coating variability in a rotary batch seed coater, Chem. Eng. Res. Des., 2017, 120, 92-101.

78 Y. Qiu, D. R. Myers and W. A. Lam, The biophysics and mechanics of blood from a materials perspective, Nat. Rev. Mater., 2019, 4, 294-311.

79 M. R. I. Sarder, M. M. Sarker and S. K. Saha, Cryopreservation of sperm of an indigenous endangered fish species Nandus nandus (Hamilton, 1822) for ex situ conservation, Cryobiology, 2012, 65, 202-209.

80 P. Alivisatos, The use of nanocrystals in biological detection, Nat. Biotechnol., 2004, 22, 47-52.

81 Y. Li, L. Zhao, Y. Yao and X. Guo, Single-molecule nanotechnologies: An evolution in the biological dynamics detection, ACS Appl. Bio Mater., 2020, 3, 68-85.

82 Y. Yi, W. Liao, Q. Zhao and X. Lu, Separation and detection of tryptophan metabolites in biological samples, Chin. J. Chromatogr., 1999, 17, 158-161.

83 L. Calvo and J. Casas, Sterilization of biological weapons in technical clothing and sensitive material by high-pressure $\mathrm{CO}_{2}$ and water, Ind. Eng. Chem. Res., 2018, 57, 4680-4687.

84 G. Zhao, C. He, D. Kumar, J. P. Hooper, G. H. Imler, D. A. Parrish and M. S. Jean'ne, 1,3,5-Triiodo-2,4,6-trinitrobenzene (TITNB) from benzene: Balancing performance and high thermal stability of functional energetic materials, Chem. Eng. J., 2019, 378, 122119.

85 M. Bray, Defense against filoviruses used as biological weapons, Antiviral Res., 2003, 57, 53-60.

86 A. J. Russell, J. A. Berberich, G. r. F. Drevon and R. R. Koepsel, Biomaterials for mediation of chemical and biological warfare agents, Annu. Rev. Biomed. Eng., 2003, 5, 1-27.

87 G. Zhao, C. He, D. Kumar, J. P. Hooper, G. H. Imler, D. A. Parrish and M. S. Jean'ne, Functional energetic biocides by coupling of energetic and biocidal polyiodo building blocks, Chem. Eng. J., 2019, 368, 244-251.

88 I. Van der Lubben, J. Verhoef, G. Borchard and H. Junginger, Chitosan for mucosal vaccination, Adv. Drug Delivery Rev., 2001, 52, 139-144.

89 B. Pulendran and R. Ahmed, Immunological mechanisms of vaccination, Nat. Immunol., 2011, 12, 509.

90 S. P. Sullivan, D. G. Koutsonanos, M. del Pilar Martin, J. W. Lee, V. Zarnitsyn, S.-O. Choi, N. Murthy, R. W. Compans, I. Skountzou and M. R. Prausnitz, Dissolving polymer 
microneedle patches for influenza vaccination, Nat. Med., 2010, 16, 915.

91 L. O. De Serrano and D. J. Burkhart, Liposomal vaccine formulations as prophylactic agents: design considerations for modern vaccines, J. Nanobiotechnol., 2017, 15, 83.

92 M. A. Islam, E. K. Reesor, Y. Xu, H. R. Zope, B. R. Zetter and J. Shi, Biomaterials for mRNA delivery, Biomater. Sci., 2015, 3, 1519-1533.

93 P. S. Kowalski, A. Rudra, L. Miao and D. G. Anderson, Delivering the Messenger: Advances in Technologies for Therapeutic mRNA Delivery, Mol. Ther., 2019, 27, 710-728.

94 N. B. Tsui, E. K. Ng and Y. M. Lo, Stability of endogenous and added RNA in blood specimens, serum, and plasma, Clin. Chem., 2002, 48, 1647-1653.

95 J. Houseley and D. Tollervey, The many pathways of RNA degradation, Cell, 2009, 136, 763-776.

96 J. Zhang, H. Zeng, J. Gu, H. Li, L. Zheng and Q. Zou, Progress and Prospects on Vaccine Development against SARS-CoV-2, Vaccines, 2020, 8, 153.

97 T. T. Le, Z. Andreadakis, A. Kumar, R. G. Román, S. Tollefsen, M. Saville and S. Mayhew, The COVID-19 vaccine development landscape, Nat. Rev. Drug Discovery, 2020, 19, 305-306.

$98 \mathrm{~J}$. Cohen, Vaccine designers take first shots at COVID-19, Science, 2020, 368, 14-16.

99 J. E. Dahlman, C. Barnes, O. Khan, A. Thiriot, S. Jhunjunwala, T. E. Shaw, Y. Xing, H. B. Sager, G. Sahay, L. Speciner, A. Bader, R. L. Bogorad, H. Yin, T. Racie, Y. Dong, S. Jiang, D. Seedorf, A. Dave, K. S. Sandu, M. J. Webber, T. Novobrantseva, V. M. Ruda, A. K. R. Lytton-Jean, C. G. Levins, B. Kalish, D. K. Mudge, M. Perez, L. Abezgauz, P. Dutta, L. Smith, K. Charisse, M. W. Kieran, K. Fitzgerald, M. Nahrendorf, D. Danino, R. M. Tuder, U. H. von Andrian, A. Akinc, A. Schroeder, D. Panigrahy, V. Kotelianski, R. Langer and D. G. Anderson, In vivo endothelial siRNA delivery using polymeric nanoparticles with low molecular weight, Nat. Nanotechnol., 2014, 9, 648-655.

100 X. Han, S. Shen, Q. Fan, G. Chen, E. Archibong, G. Dotti, Z. Liu, Z. Gu and C. Wang, Red blood cell-derived nanoerythrosome for antigen delivery with enhanced cancer immunotherapy, Sci. Adv., 2019, 5, eaaw6870.

101 A. Tan, H. De La Pena and A. M. Seifalian, The application of exosomes as a nanoscale cancer vaccine, Int. J. Nanomed., 2010, 5, 889-900.

102 Y. C. Kim, J. H. Park and M. R. Prausnitz, Microneedles for drug and vaccine delivery, Adv. Drug Delivery Rev., 2012, 64, 1547-1568.

103 L. C. Burnett, G. Lunn and R. Coico, Biosafety: guidelines for working with pathogenic and infectious microorganisms, Curr. Protoc. Microbiol., 2009, 13, 1A.1.1-1A.1.14.

104 Y.-T. Yeh, K. Gulino, Y. Zhang, A. Sabestien, T.-W. Chou, B. Zhou, Z. Lin, I. Albert, H. Lu and V. Swaminathan, A rapid and label-free platform for virus capture and identification from clinical samples, Proc. Natl. Acad. Sci. U. S. A., 2020, 117, 895-901.

105 F. Sun, A. Ganguli, J. Nguyen, R. Brisbin, K. Shanmugam, D. L. Hirschberg, M. B. Wheeler, R. Bashir, D. M. Nash and
B. T. Cunningham, Smartphone-based multiplex 30-minute nucleic acid test of live virus from nasal swab extract, $L a b$ Chip, 2020, 20, 1621-1627.

106 F. Zang, Z. Su, L. Zhou, K. Konduru, G. Kaplan and S. Y. Chou, Ultrasensitive Ebola Virus Antigen Sensing via 3D Nanoantenna Arrays, Adv. Mater., 2019, 31, e1902331.

107 Y.-T. Yeh, Y. Tang, A. Sebastian, A. Dasgupta, N. PereaLopez, I. Albert, H. Lu, M. Terrones and S.-Y. Zheng, Tunable and label-free virus enrichment for ultrasensitive virus detection using carbon nanotube arrays, Sci. Adv., 2016, 2, e1601026.

108 P. Zhu, Y. Chen and J. Shi, Nanoenzyme-augmented cancer sonodynamic therapy by catalytic tumor oxygenation, ACS Nano, 2018, 12, 3780-3795.

109 L. Wang, L. Miao, H. Yang, J. Yu, Y. Xie, L. Xu and Y. Song, A novel nanoenzyme based on Fe3O4 nanoparticles@ thionine-imprinted polydopamine for electrochemical biosensing, Sens. Actuators, B, 2017, 253, 108-114.

110 S. R. Ahmed, J. C. Corredor, E. Nagy and S. Neethirajan, Amplified visual immunosensor integrated with nanozyme for ultrasensitive detection of avian influenza virus, Nanotheranostics, 2017, 1, 338-345.

111 D. Duan, K. Fan, D. Zhang, S. Tan, M. Liang, Y. Liu, J. Zhang, P. Zhang, W. Liu, X. Qiu, G. P. Kobinger, G. F. Gao and $\mathrm{X}$. Yan, Nanozyme-strip for rapid local diagnosis of Ebola, Biosens. Bioelectron., 2015, 74, 134-141.

112 A. Giraud, I. Matic, M. Radman, M. Fons and F. Taddei, Mutator bacteria as a risk factor in treatment of infectious diseases, Antimicrob. Agents Chemother., 2002, 46, 863-865.

113 A. W. Stableforth and I. A. Galloway, Infectious diseases of animals. Diseases due to Bacteria, Butterworths Scientific Publications, London, 1959, vol. 2.

114 M. Xiao, K. Zou, L. Li, L. Wang, Y. Tian, C. Fan and H. Pei, Stochastic DNA Walkers in Droplets for Super-Multiplexed Bacterial Phenotype Detection, Angew. Chem., Int. Ed., 2019, 58, 15448-15454.

115 Y. Hu, Y. He, Y. Han, Y. Ge, G. Song and J. Zhou, Determination of the activity of alkaline phosphatase based on aggregationinduced quenching of the fluorescence of copper nanoclusters, Microchim. Acta, 2019, 186, 5.

116 X. Gu, G. Zhang and D. Zhang, A new ratiometric fluorescence detection of heparin based on the combination of the aggregation-induced fluorescence quenching and enhancement phenomena, Analyst, 2012, 137, 365-369.

117 Y. Hong, J. W. Lam and B. Z. Tang, Aggregation-induced emission, Chem. Soc. Rev., 2011, 40, 5361-5388.

118 A. Qin, J. W. Lam and B. Z. Tang, Luminogenic polymers with aggregation-induced emission characteristics, Prog. Polym. Sci., 2012, 37, 182-209.

119 X. He, Y. Yang, Y. Guo, S. Lu, Y. Du, J.-J. Li, X. Zhang, N. L. Leung, Z. Zhao and G. Niu, Phage-Guided Targeting, Discriminative Imaging, and Synergistic Killing of Bacteria by AIE Bioconjugates, J. Am. Chem. Soc., 2020, 142, 3959-3969.

120 A. Panigrahi, V. N. Are, S. Jain, D. Nayak, S. Giri and T. K. Sarma, Cationic Organic Nanoaggregates as AIE 
Luminogens for Wash-Free Imaging of Bacteria and BroadSpectrum Antimicrobial Application, ACS Appl. Mater. Interfaces, 2020, 12, 5389-5402.

121 M. Kang, R. T. Kwok, J. Wang, H. Zhang, J. W. Lam, Y. Li, P. Zhang, H. Zou, X. Gu and F. Li, A multifunctional luminogen with aggregation-induced emission characteristics for selective imaging and photodynamic killing of both cancer cells and Gram-positive bacteria, J. Mater. Chem. B, 2018, 6, 3894-3903.

122 M. Wu, G. Qi, X. Liu, Y. Duan, J. Liu and B. Liu, BioOrthogonal AIEgen for Specific Discrimination and Elimination of Bacterial Pathogens via Metabolic Engineering, Chem. Mater., 2019, 32, 858-865.

123 T. Zhou, R. Hu, L. Wang, Y. Qiu, G. Zhang, Q. Deng, H. Zhang, P. Yin, B. Situ and C. Zhan, AIE conjugated polymer with ultra-strong ROS generation ability and great biosafety for efficient therapy of bacterial infection, Angew. Chem., Int. Ed., 2020, 55, 16704-16710.

124 V. Müller, J. M. Sousa, H. C. Koydemir, M. Veli, D. Tseng, L. Cerqueira, A. Ozcan, N. F. Azevedo and F. Westerlund, Identification of pathogenic bacteria in complex samples using a smartphone based fluorescence microscope, $R S C$ Adv., 2018, 8, 36493-36502.

125 R. Guo, C. McGoverin, S. Swift and F. Vanholsbeeck, A rapid and low-cost estimation of bacteria counts in solution using fluorescence spectroscopy, Anal. Bioanal. Chem., 2017, 409, 3959-3967.

126 X. Huang, Y. Yang, J. Shi, H. T. Ngo, C. Shen, W. Du and Y. Wang, High-Internal-Phase Emulsion Tailoring Polymer Amphiphilicity towards an Efficient NIR-Sensitive Bacteria Filter, Small, 2015, 11, 4876-4883.

127 S. Deshmukh, S. Patil, S. Mullani and S. Delekar, Silver nanoparticles as an effective disinfectant: a review, Mater. Sci. Eng., C, 2019, 97, 954-965.

128 F. Huang, Y. Gao, Y. Zhang, T. Cheng, H. Ou, L. Yang, J. Liu, L. Shi and J. Liu, Silver-decorated polymeric micelles combined with curcumin for enhanced antibacterial activity, ACS Appl. Mater. Interfaces, 2017, 9, 16880-16889.

129 A. Scalbert, Antimicrobial properties of tannins, Phytochemistry, 1991, 30, 3875-3883.

130 W. F. Broekaert, B. P. Cammue, M. F. De Bolle, K. Thevissen, G. W. De Samblanx, R. W. Osborn and K. Nielson, Antimicrobial peptides from plants, Crit. Rev. Plant Sci., 1997, 16, 297-323.

131 G. Qing, X. Zhao, N. Gong, J. Chen, X. Li, Y. Gan, Y. Wang, Z. Zhang, Y. Zhang and W. Guo, Thermo-responsive triplefunction nanotransporter for efficient chemo-photothermal therapy of multidrug-resistant bacterial infection, Nat. Commun., 2019, 10, 1-12.

132 H. Zheng, Z. Ji, K. R. Roy, M. Gao, Y. Pan, X. Cai, L. Wang, W. Li, C. H. Chang and C. Kaweeteerawat, Engineered Graphene Oxide Nanocomposite Capable of Preventing the Evolution of Antimicrobial Resistance, ACS Nano, 2019, 13, 11488-11499.

133 M. L. Ranney, V. Griffeth and A. K. Jha, Critical supply shortages - the need for ventilators and personal protective equipment during the Covid-19 pandemic, N. Engl. J. Med., 2020, 382, 41-44.

$134 \mathrm{~W}$. H. Organization, Rational use of personal protective equipment for coronavirus disease (COVID-19) and considerations during severe shortages: interim guidance, 6 April 2020, World Health Organization, 2020.

135 H. Zhong, Z. Zhu, J. Lin, C. F. Cheung, V. L. Lu, F. Yan, C.-Y. Chan and G. Li, Reusable and Recyclable Graphene Masks with Outstanding Superhydrophobic and Photothermal Performances, ACS Nano, 2020, DOI: 10.1021/ acsnano.0c02250.

136 A. Konda, A. Prakash, G. A. Moss, M. Schmoldt, G. D. Grant and S. Guha, Aerosol filtration efficiency of common fabrics used in respiratory cloth masks, ACS Nano, 2020, DOI: 10.1021 /acsnano.0c03252.

137 P. Li, J. Li, X. Feng, J. Li, Y. Hao, J. Zhang, H. Wang, A. Yin, J. Zhou and X. Ma, Metal-organic frameworks with photocatalytic bactericidal activity for integrated air cleaning, Nat. Commun., 2019, 10, 1-10.

138 G. Liu, J. Nie, C. Han, T. Jiang, Z. Yang, Y. Pang, L. Xu, T. Guo, T. Bu and C. Zhang, Self-powered electrostatic adsorption face mask based on a triboelectric nanogenerator, ACS Appl. Mater. Interfaces, 2018, 10, 7126-7133.

139 V. Parthasarathi and G. Thilagavathi, Development of plasma enhanced antiviral surgical gown for healthcare workers, Fashion Text., 2015, 2, 4-16.

140 A. Nel, Air pollution-related illness: effects of particles, Science, 2005, 308, 804-806.

141 B. Brunekreef and S. T. Holgate, Air pollution and health, Lancet, 2002, 360, 1233-1242.

142 M. S. Shafeeyan, W. M. A. W. Daud, A. Houshmand and A. Shamiri, A review on surface modification of activated carbon for carbon dioxide adsorption, J. Anal. Appl. Pyrolysis, 2010, 89, 143-151.

143 Z. Feng, Z. Long and T. Yu, Filtration characteristics of fibrous filter following an electrostatic precipitator, J. Electrost., 2016, 83, 52-62.

144 D. Provenzano, Y. J. Rao, K. Mitic, S. N. Obaid, J. Berger, S. Goyal and M. H. Loew, Alternative Qualitative Fit Testing Method for N95 Equivalent Respirators in the Setting of Resource Scarcity at the George Washington University, 2020, medRxiv, DOI: 10.1101/2020.04.06.20055368.

145 S. Au, C. Gomersall, P. Leung and P. Li, A randomised controlled pilot study to compare filtration factor of a novel non-fit-tested high-efficiency particulate air (HEPA) filtering facemask with a fit-tested N95 mask, J. Hosp. Infect., 2010, 76, 23-25.

146 M. Cai, H. Li, S. Shen, Y. Wang and Q. Yang, Customized design and 3D printing of face seal for an N95 filtering facepiece respirator, J. Occup. Environ. Hyg., 2018, 15, 226-234.

147 A. Deplazes-Zemp, 'Genetic resources', an analysis of a multifaceted concept, Biol. Conserv., 2018, 222, 86-94.

$148 \mathrm{~T}$. Abramishvili and N. Chkhaidze, The role of bioagents in protection of wheat genetic resources, Commun. Agric. Appl. Biol. Sci., 2009, 74, 401-406. 
149 R. Scholl, L. Rivero-Lepickas and D. Crist, Arabidopsis Protocols, Springer, 1998, pp. 1-12.

150 C. Walters, P. Berjak, N. Pammenter, K. Kennedy and P. Raven, PLANT SCIENCE: Preservation of Recalcitrant Seeds, Science, 2013, 339, 915-916.

151 K. Semagn, Molecular Plant Taxonomy, Springer, 2014, pp. 53-67.

152 F. S. Chiou, C. Y. Pai, Y. P. P. Hsu, C. W. Tsai and C. H. Yang, Extraction of human DNA for PCR from chewed residues of betel quid using a novel "PVP/CTAB" method, J. Forensic Sci., 2001, 46, 1174-1179.

153 M. Guamán-Balcázar, A. Montes, C. Pereyra and E. M. de la Ossa, Production of submicron particles of the antioxidants of mango leaves/PVP by supercritical antisolvent extraction process, J. Supercrit. Fluids, 2019, 143, 294-304.

154 L. Dennany, R. J. Förster, B. White, M. Smyth and J. F. Rusling, Direct electrochemiluminescence detection of oxidized DNA in ultrathin films containing $\left[\mathrm{Os}(\mathrm{bpy})_{2}(\mathrm{PVP})_{10}\right]^{2+}, \mathrm{J} . \mathrm{Am}$. Chem. Soc., 2004, 126, 8835-8841.

155 I.-K. Park, J.-E. Ihm, Y. Park, Y. Choi, S. Kim, W. Kim, T. Akaike and C. Cho, Galactosylated chitosan (GC)-graftpoly(vinyl pyrrolidone) (PVP) as hepatocyte-targeting DNA carrier: preparation and physicochemical characterization of GC-graft-PVP/DNA complex (1), J. Controlled Release, 2003, 86, 349-359.

156 X. Yu, Q. Luo, K. Huang, G. Yang and G. He, Prospecting for microelement function and biosafety assessment of transgenic cereal plants, Frontiers in plant, Science, 2018, 9, 326.

157 G. N. Y. Lemgo, S. Sabbadini, T. Pandolfini and B. Mezzetti, Biosafety considerations of RNAi-mediated virus resistance in fruit-tree cultivars and in rootstock, Transgenic Res., 2013, 22, 1073-1088.

158 A. T. Zvinavashe, E. Lim, H. Sun and B. Marelli, A bioinspired approach to engineer seed microenvironment to boost germination and mitigate soil salinity, Proc. Natl. Acad. Sci. U. S. A., 2019, 116, 25555-25561.

159 P. Tseng, B. Napier, S. Zhao, A. N. Mitropoulos, M. B. Applegate, B. Marelli, D. L. Kaplan and F. G. Omenetto, Directed assembly of bio-inspired hierarchical materials with controlled nanofibrillar architectures, Nat. Nanotechnol., 2017, 12, 474.

160 A. Matsumoto, A. Lindsay, B. Abedian and D. L. Kaplan, Silk fibroin solution properties related to assembly and structure, Macromol. Biosci., 2008, 8, 1006-1018.

161 B. Marelli, M. Brenckle, D. L. Kaplan and F. G. Omenetto, Silk fibroin as edible coating for perishable food preservation, Sci. Rep., 2016, 6, 25263.

162 H.-J. Jin and D. L. Kaplan, Mechanism of silk processing in insects and spiders, Nature, 2003, 424, 1057-1061.

163 E. Porcu, R. Fabbri, R. Seracchioli, P. M. Ciotti, O. Magrini and C. Flamigni, Birth of a healthy female after intracytoplasmic sperm injection of cryopreserved human oocytes, Fertil. Steril., 1997, 68, 724-726.

164 Y. Wang, X. Yao, S. Wu, Q. Li, J. Lv, J. Wang and L. Jiang, Bioinspired solid organogel materials with a regenerable sacrificial alkane surface layer, Adv. Mater., 2017, 29, 1700865.

165 I. Jarić, T. Heger, F. C. Monzon, J. M. Jeschke, I. Kowarik, K. R. McConkey, P. Pyšek, A. Sagouis and F. Essl, Crypticity in biological invasions, Trends Eol. Evol., 2019, 34, 291-302.

166 T. M. Blackburn, P. Pyšek, S. Bacher, J. T. Carlton, R. P. Duncan, V. C. Jarošík, J. R. Wilson and D. M. Richardson, A proposed unified framework for biological invasions, Trends Ecol. Evol., 2011, 26, 333-339.

167 A. Tayeh, R. A. Hufbauer, A. Estoup, V. Ravigné, L. A. Frachon and B. Facon, Biological invasion and biological control select for different life histories, Nat. Commun., 2015, 6, 1-5.

168 C. L. Bellard, J.-F. O. Rysman, B. Leroy, C. Claud and G. M. Mace, A global picture of biological invasion threat on islands, Nat. Ecol. Evol., 2017, 1, 1862-1869.

169 D. Simberloff, J.-L. Martin, P. Genovesi, V. Maris, D. A. Wardle, J. Aronson, F. Courchamp, B. Galil, E. GarcíaBerthou and M. Pascal, Impacts of biological invasions: what's what and the way forward, Trends Ecol. Evol., 2013, 28, 58-66.

170 L. P. Xu, Y. Chen, G. Yang, W. Shi, B. Dai, G. Li, Y. Cao, Y. Wen, X. Zhang and S. Wang, Ultratrace DNA detection based on the condensing-enrichment effect of superwettable microchips, Adv. Mater., 2015, 27, 6878-6884.

171 H. Y. El-Kassas and M. G. Ghobrial, Biosynthesis of metal nanoparticles using three marine plant species: anti-algal efficiencies against “Oscillatoria simplicíssima”, Environ. Sci. Pollut. Res., 2017, 24, 7837-7849.

172 G. Rajakumar and A. A. Rahuman, Larvicidal activity of synthesized silver nanoparticles using Eclipta prostrata leaf extract against filariasis and malaria vectors, Acta Trop., 2011, 118, 196-203.

173 Y. Y. Song, J. J. Zhou, J. B. Fan, W. Z. Zhai, J. X. Meng and S. T. Wang, Hydrophilic/Oleophilic Magnetic Janus Particles for the Rapid and Efficient Oil-Water Separation, Adv. Funct. Mater., 2018, 28, 1802493.

174 C. Ren, J. Shen, F. Chen and H. Zeng, Rapid RoomTemperature Gelation of Crude Oils by a Wetted Powder Gelator, Angew. Chem., Int. Ed., 2017, 56, 3847-3851.

175 X.-J. Ju, S.-B. Zhang, M.-Y. Zhou, R. Xie, L. Yang and L.-Y. $\mathrm{Chu}$, Novel heavy-metal adsorption material: ion-recognition $\mathrm{P}($ NIPAM-co-BCAm) hydrogels for removal of lead(II) ions, J. Hazard. Mater., 2009, 167, 114-118.

176 M. Lian, Q. Feng, L. Wang, L. Niu, Z. Zhao, X. Li and $\mathrm{Z}$. Zhang, Highly effective immobilization of $\mathrm{Pb}$ and $\mathrm{Cd}$ in severely contaminated soils by environment-compatible, mercapto-functionalized reactive nanosilica, J. Cleaner Prod., 2019, 235, 583-589.

177 L. R. R. Souza, L. C. Pomarolli and M. A. M. S. da Veiga, From classic methodologies to application of nanomaterials for soil remediation: an integrated view of methods for decontamination of toxic metal(oid)s, Environ. Sci. Pollut. Res., 2020, 27, 10205-10227.

178 Z. Peng, X. Liu, W. Zhang, Z. Zeng, Z. Liu, C. Zhang, Y. Liu, B. Shao, Q. Liang and W. Tang, Advances in the application, 
toxicity and degradation of carbon nanomaterials in environment: a review, Environ. Int., 2020, 134, 105298.

179 K. Kerwat, S. Becker, H. Wulf and D. Densow, Biological weapons, Dtsch. Med. Wochenschr., 2010, 135, 1612-1616.

180 A. Akçali, Viruses as biological weapons, Mikrobiyol. Bul., 2005, 39, 383-397.

181 G. Pappas, P. Panagopoulou, L. Christou and N. Akritidis, Biological weapons, Cell. Mol. Life Sci., 2006, 63, 2229-2236.

182 K. Luan, R. Meng, C. Shan, J. Cao, J. Jia, W. Liu and Y. Tang, Terbium Functionalized Micelle Nanoprobe for Ratiometric Fluorescence Detection of Anthrax Spore Biomarker, Anal. Chem., 2018, 90, 3600-3607.

183 D. J. Bower, R. J. Hart, P. A. Matthews and M. E. Howden, Nonprotein neurotoxins, Clin. Toxicol., 1981, 18, 813-863.

184 J. Segura-Aguilar and R. M. Kostrzewa, Neurotoxins and neurotoxicity mechanisms. An overview, Neurotoxic. Res., 2006, 10, 263-285.

185 B. Poulain, La neurotoxine botulinique, Rev. Neurol., 2010, 166, 7-20.

186 P. Zhang, E. J. Liu, C. Tsao, S. A. Kasten, M. V. Boeri, T. L. Dao, S. J. DeBus, C. L. Cadieux, C. A. Baker and T. C. Otto, Nanoscavenger provides long-term prophylactic protection against nerve agents in rodents, Sci. Transl. Med., 2019, 11, eaau7091.

187 S. Y. Kwak, T. T. S. Lew, C. J. Sweeney, V. B. Koman, M. H. Wong, K. Bohmert-Tatarev, K. D. Snell, J. S. Seo, N. H. Chua and M. S. Strano, Chloroplast-selective gene delivery and expression in planta using chitosan-complexed single-walled carbon nanotube carriers, Nat. Nanotechnol., 2019, 14, 447-455.

188 P. Wang, F.-J. Zhao and P. M. Kopittke, Engineering crops without genome integration using nanotechnology, Trends Plant Sci., 2019, 24, 574-577.

189 M. S. Brewer, G. K. Sprouls and C. Russon, Consumer attitudes toward food safety issues, J. Food Saf., 1994, 14, 63-76.

190 S. Miles, M. Brennan, S. Kuznesof, M. Ness, C. Ritson and L. J. Frewer, Public worry about specific food safety issues, Br. Food J., 2004, 92, 9-22.

191 A. R. Shalaby, Significance of biogenic amines to food safety and human health, Food Res. Int., 1996, 29, 675-690.

192 A. Röhr, K. Lüddecke, S. Drusch, M. J. Müller and R. Alvensleben, Food quality and safety-consumer perception and public health concern, Food Control, 2005, 16, 649-655.

193 S. P. Oliver, B. M. Jayarao and R. A. Almeida, Foodborne pathogens in milk and the dairy farm environment: food safety and public health implications, Foodbourne Pathog. Dis., 2005, 2, 115-129.

194 J.-C. Ogier and P. Serror, Safety assessment of dairy microorganisms: the Enterococcus genus, Int. J. Food Microbiol., 2008, 126, 291-301.
195 D. Rodríguez-Lázaro, B. Lombard, H. Smith, A. Rzezutka, M. D'Agostino, R. Helmuth, A. Schroeter, B. Malorny, A. Miko and B. Guerra, Trends in analytical methodology in food safety and quality: monitoring microorganisms and genetically modified organisms, Trends Food Sci. Technol., 2007, 18, 306-319.

196 S. He, W. Xie, W. Zhang, L. Zhang, Y. Wang, X. Liu, Y. Liu and C. Du, Multivariate qualitative analysis of banned additives in food safety using surface enhanced Raman scattering spectroscopy, Spectrochim. Acta, Part A, 2015, 137, 1092-1099.

197 L. Tollefson, Monitoring adverse reactions to food additives in the US Food and Drug Administration, Regul. Toxicol. Pharmacol., 1988, 8, 438-446.

198 F. Arduini, S. Cinti, V. Scognamiglio and D. Moscone, Nanomaterials in electrochemical biosensors for pesticide detection: advances and challenges in food analysis, Microchim. Acta, 2016, 183, 2063-2083.

199 C. K. Winter and E. A. Jara, Pesticide food safety standards as companions to tolerances and maximum residue limits, J. Integr. Agric., 2015, 14, 2358-2364.

200 K. Weidemaier, E. Carruthers, A. Curry, M. Kuroda, E. Fallows, J. Thomas, D. Sherman and M. Muldoon, Realtime pathogen monitoring during enrichment: a novel nanotechnology-based approach to food safety testing, Int. J. Food Microbiol., 2015, 198, 19-27.

201 A. Mousavi, M. Sarhadi, S. Fawcett, S. Bowles and M. York, Tracking and traceability solution using a novel material handling system, Innovative Food Sci. Emerging Technol., 2005, 6, 91-105.

202 T. McHugh and E. Senesi, Apple wraps: A novel method to improve the quality and extend the shelf life of fresh-cut apples, J. Food Sci., 2000, 65, 480-485.

203 D. Liu and N. Gu, Nanomaterials for fresh-keeping and sterilization in food preservation, Recent Pat. Food, Nutr. Agric., 2009, 1, 149-154.

204 S. Tang, T. Qi, D. Xia, M. Xu, M. Xu, A. Zhu, W. Shen and H. K. Lee, Smartphone Nanocolorimetric Determination of Hydrogen Sulfide in Biosamples after Silver-Gold CoreShell Nanoprism-Based Headspace Single-Drop Microextraction, Anal. Chem., 2019, 91, 5888-5895.

205 K. A. Doherty, J. G. Carton, A. Norman, T. McCaul, B. Twomey and K. T. Stanton, A thermal control surface for the Solar Orbiter, Acta Astronaut., 2015, 117, 430-439.

206 T. Ghidini, Materials for space exploration and settlement, Nat. Mater., 2018, 17, 846-850.

207 S. R. Zavada, N. R. McHardy, K. L. Gordon and T. F. Scott, Rapid, Puncture-Initiated Healing via Oxygen-Mediated Polymerization, ACS Macro Lett., 2015, 4, 819-824. 